\title{
Cronología de la regeneración del bosque tropical seco en Santa Rosa, Guanacaste, Costa Rica. II. La vegetación en relación con el suelo
}

\author{
Jorge A. Leiva ${ }^{1}$, Oscar J. Rocha ${ }^{2}$, Rafael Mata ${ }^{1} \&$ Marco V. Gutiérrez-Soto ${ }^{3 *}$ \\ 1. Universidad de Costa Rica, Centro de Investigaciones Agronómicas, 11503-2060, San José, Costa Rica; \\ jorge.leivasanabria@ucr.ac.cr \\ 2. Department of Biological Sciences, Kent State University, Ohio, USA; orocha@kent.edu \\ 3. Autor para correspondencia: Universidad de Costa Rica, Estación Experimental Fabio Baudrit, Apdo. 183-4050 \\ Alajuela, Costa Rica; Teléfono: (506) 2433-9111, Fax: (506) 2433-9086; surdo26@racsa.co.cr
}

\author{
Recibido 15-V-2008. Corregido 13-I-2009. Aceptado 10-II-2009.
}

\begin{abstract}
Chronology of tropical dry forest regeneration in Santa Rosa, Guanacaste, Costa Rica. II. Vegetation in relation to the soil. Tropical dry forest (TDF) succession was monitored in Santa Rosa, Costa Rica. We analyzed the effect of soil type on forest structure and diversity. Eight seasonally-dry TDF sites located along a successional chrono-sequence $(10,15,20,40,60$ and $>100$ years) were examined in relation to 17 soil pedons and six soil orders. Soils had moderate to high fertility and were classified as Entisols and Vertisols, although Mollisols, Alfisols, Inceptisols and Ultisols were also present. One-hundred and thirty $500 \mathrm{~m}^{2}$ plots were established, 20 plots in secondary and 10 plots in mature TDFsites. Diameter at breast height (dbh) and total tree height were measured for saplings ( $\mathrm{dbh} \geq 1$ and $<5 \mathrm{~cm}$ ), shrubs and trees $(\mathrm{dbh} \geq 5 \mathrm{~cm})$. With the exception of two sites (40 and 60 years), soil type did not have significant effects on forest structure. However, tree diversity measured with Shannon-Wiener's H' and Fisher's $\alpha$ rarefaction curves, showed substantial differences among soil types, which became accentuated in mature forests. This pattern might be explained by non-random distributions of TDF trees, the scale of the study, the plot shape, and the use of systematic sampling designs. Low-fertility sites in general had higher species richness, consistent with idea that more restrictive soils reduce competition among trees and allow co-existence of species with contrasting growth rates. Changes in soil properties along a chrono-sequence of Entisols indicated that trees may experience more severe water stress as succession progresses, which may require adjustments in biomass allocation and phenological behavior of the dominant species. Our results suggest that edaphic specialization is more pronounced in mature TDF forests, and that most TDF trees are generalists in relation to soil type, highly tolerant to site heterogeneity, and show little physiological specializations in response to edaphic heterogeneity. Rev. Biol. Trop. 57 (3): 817-836. Epub 2009 September 30.
\end{abstract}

Keywords: tropical soils, Santa Rosa National Park, soil chemical properties, soil physical properties, tropical dry forest, succession, secondary forests, mature forests.

Los bosques tropicales estacionalmente secos (BTS) se presentan en áreas donde la temperatura promedio oscila entre $24-28{ }^{\circ} \mathrm{C}$, la precipitación anual varía entre $1000-2000 \mathrm{~mm}$ y la relación entre la evapotranspiración y la precipitación anuales es $>1$ (Holdridge 1978). La principal característica climática del BTS, que define la mayoría de sus procesos ecológicos, es la incidencia de un periodo de sequía anual (Murphy \& Lugo 1995). El BTS era uno de los ecosistemas boscosos de mayor extensión en Mesoamérica (Janzen 1986), pero en la actualidad es considerado el ecosistema más amenazado del Neotrópico (Janzen 1988). En Santa Rosa, Costa Rica, las haciendas ganaderas y la extracción maderera han sido las principales causas de la pérdida de esta zona de vida (Maas 1995, Murphy \& Lugo 1995).

En la actualidad, los BTS constituyen mosaicos de fragmentos boscosos secundarios 
en diferentes estados de regeneración (Kramer 1997). La conservación y la restauración del BTS dependerán en última instancia de la operación de la sucesión ecológica. Asociado a los suelos formados sobre las mesetas ignimbríticas de Bagaces y Liberia (Leiva et al. 2009) se ha generado un paisaje fragmentado en el que parches de BTS maduro alternan con repastos ganaderos, sitios abandonados, y áreas cultivadas, que provee un sistema apropiado para el estudio de la regeneración del BTS. Esta recuperación puede ocurrir en periodos relativamente cortos (Chazdon 2003, Ruiz et al. 2005), pero en contraste con los bosques templados (Bazzaz 1979) y los ecosistemas tropicales lluviosos (Bazzaz \& Pickett 1980, Mulkey et al. 1996), el conocimiento de los procesos sucesionales en el BTS es considerablemente menor (Ewel 1977, 1980, Stemmermann e Ihsle 1993).

La sucesión del BTS es controlada en gran medida por la historia del uso de la tierra y la influencia de la vegetación remanente luego del abandono del sitio. La regeneración boscosa en los BTS es seriamente retrasada por perturbaciones severas como el fuego y la sequía (Ewel 1977, 1980), y tiende a ocurrir más lentamente que en los bosques lluviosos. La dinámica de los claros y el banco de semillas del suelo son de menor importancia en la regeneración del BTS (Rico-Gray \& García-Franco 1992), y la reproducción vegetativa es frecuente en estados sucesionales tempranos (Ewel 1977). La diversidad de especies y el número de especies por área son menores en los BTS que en los bosques lluviosos, y el crecimiento en altura ocurre en pulsos estacionales, el dosel resultante es irregular, de menor altura y menos denso. A estos efectos debe sumarse la alta heterogeneidad edáfica espacial (Leiva et al. 2009), los que conjuntamente pueden resultar en múltiples trayectorias sucesionales (Ewel 1980, Mesquita 2001, Chazdon 2003). Aunque varios estudios indican que el suelo influencia la estructura, la diversidad y la abundancia de muchas especies de árboles tropicales (Huston 1980, Clark et al. 1999), las variaciones edáficas locales y sus efectos sobre la ruta sucesional han recibido la menor atención.
La vegetación del BTS de Santa Rosa es dominada por especies deciduas que pierden el follaje durante la estación seca, las cuales coexisten con un número importante de especies siempre-verdes y brevi-deciduas que difieren en el momento y la duración de la defoliación (Holbrook et al. 1995, Eamus \& Prior 2001). Los sitios sucesionales jóvenes recientemente quemados son dominados por especies pioneras cuyo desarrollo puede ser propiciado por el fuego; los troncos con tallos múltiples son frecuentes (Ewel 1977). Estas pioneras precoces y de rápido crecimiento, hábitos fenológicos variados, altos requerimientos de luz, y semillas dispersadas por el viento, pueden alcanzar alturas de 15-25 m y longevidades que varían entre 50 y varios cientos de años (Janzen 1988). Algunas pioneras de menor porte son progresivamente reemplazadas por formas de vida más diversas y de mayor altura (Stemmermann \& Ihsle 1993), algunas de las cuales toleran bien la sombra. Los bosques maduros son diversos y con un alto grado de endemismo, y pueden contener abundantes especies brevideciduas y siempre-verdes de crecimiento más lento (Eamus \& Prior 2001), que mantienen condiciones ambientales más favorables en el sotobosque, incluso durante la estación seca. A pesar de que los efectos de la vegetación sobre el suelo son variados e incluyen cambios en $\mathrm{pH}$, capacidad de intercambio catiónico (CIC) y bases cambiables (Zinke 1962), cantidad y calidad de la materia orgánica (Russell et al. 2007), y contenido de nitrógeno (Smith et al. 1998), los estudios de las relaciones sueloplanta durante la sucesión son muy escasos (Lathwell \& Grove 1986, Clark 2002).

En un estudio conjunto, Leiva et al. (2009) caracterizaron las propiedades y la heterogeneidad espacial de los suelos a lo largo de una cronosecuencia sucesional del BTS en Santa Rosa, Costa Rica. Todos los suelos presentaron fertilidad adecuada. Se observaron incrementos en los macro-poros y en la conductividad hidráulica conforme la sucesión progresó, resultado de mayor actividad de las raíces, pero el agua disponible para las plantas disminuyó. En el presente estudio, analizamos el desarrollo 
estructural, la diversidad y la similitud de especies arbóreas a lo largo de una cronosecuencia sucesional (10, 15, 20, 40, 60 y >100 años) en relación con los suelos dominantes. Para esto, se examinaron cinco estadíos secundarios del BTS y tres maduros, regenerados sobre un mosaico edáfico de 17 clases de suelos, clasificadas en seis órdenes.

\section{MATERIALES Y MÉTODOS}

Localización y cronosecuencia de regeneración boscosa: Este estudio se realizó en el Parque Nacional Santa Rosa (PNSR, $10^{\circ} 50^{\prime} \mathrm{N}, 85^{\circ} \mathrm{W}$ ) y en la Hacienda Pelón de la Altura (HPA, $\left.10^{\circ} 51^{\prime} \mathrm{N}, 85^{\circ} 33^{\prime} \mathrm{W}\right)$, Liberia, Guanacaste, entre agosto del 2002 y julio del
2003. Las zonas de vida dominantes son el BTS y el bosque húmedo tropical transición a basal, con un clima marcadamente estacional y una estación seca de 3.5 a 6.5 meses, usualmente entre noviembre y abril. La precipitación media anual varía entre 1500 y $1950 \mathrm{~mm}$, y la temperatura media anual oscila entre 24 y $27.8^{\circ} \mathrm{C}$ (Holdridge 1978). Se estableció una secuencia de ocho sitios boscosos de seis edades aproximadas de sucesión $(10,15,20,40,60$ y >100 años, Cuadro 1) desde el abandono del sitio o última quema de pasturas ganaderas. Se consideró como bosque secundario la vegetación arbórea establecida sobre sitios abandonados, anteriormente usados como repastos ganaderos dominados por pastos exóticos que propician el fuego (Daubenmire 1972). Los bosques maduros correspondieron a fragmentos con una edad

\section{CUADRO 1}

Características de ocho BTS en Santa Rosa, Costa Rica. Los sitios corresponden a cinco bosques secundarios y tres bosques maduros

TABLE 1

Characteristics of eight tropical TDF in Santa Rosa, Costa Rica. The sites are five secondary forests and three mature forests

\begin{tabular}{|c|c|}
\hline $\begin{array}{c}\text { Bosque, Edad (años) } \\
\text { y zona de vida }\end{array}$ & $\begin{array}{l}\text { Clasificación de Su } \\
\text { (Orden y Subgrup }\end{array}$ \\
\hline $\begin{array}{l}\text { El Jaragual (10) } \\
\text { bh-PV }\end{array}$ & $\begin{array}{l}\text { Vertisoles y Entisoles } \\
\text { Ustic "Lithic" Epiaquerts } \\
\text { Typic "Lithic" Epiaquents }\end{array}$ \\
\hline $\begin{array}{l}\text { Las Mesas (15) } \\
\text { bs-t }\end{array}$ & $\begin{array}{l}\text { Entisoles } \\
\text { Lithic Ustorthents } \\
\text { Typic "Vertic" Epiaquents }\end{array}$ \\
\hline
\end{tabular}

$\begin{array}{ll}\text { El Príncipe (20) } & \text { Vertisoles y Entisoles } \\ \text { bh-PV } & \text { Lithic “Andic" Ustorthent } \\ & \text { Ustic Epiaquerts }\end{array}$

Los Cedros (40) Mollisoles e Inceptisoles bh-P $\nabla$

\author{
Entic Haplustolls \\ Andic Dystrustepts
}

Características generales de la vegetación

Dominancia de especies pioneras en repastos abandonados, incidencia periódica del fuego, dosel ralo de un estrato, abundancia de hierbas, presencia de vegetación remanente, arbustos de porte bajo y tallos múltiples.

Dominancia de especies pioneras en repastos abandonados, dosel ralo con parches de mayor densidad, abundancia de hierbas, presencia de árboles remantes, arbustos de porte bajo y tallos múltiples. Entre 1-2 estratos incipientes.

Dominancia de especies pioneras en repastos abandonados, dosel parcialmente cerrado con al menos 2 estratos, menor abundancia de hierbas, presencia de árboles remantes, arbustos de porte bajo y tallos múltiples.

Dosel cerrado y de mayor altura, 3 estratos bien definidos, hierbas poco abundantes, árboles adultos. 
CUADRO 1 (Continuación)

Características de ocho BTS en Santa Rosa, Costa Rica. Los sitios corresponden

a cinco bosques secundarios y tres bosques maduros

TABLE 1 (Continued)

Characteristics of eight tropical TDF in Santa Rosa, Costa Rica. The sites are

five secondary forests and three mature forests

\begin{tabular}{ll}
\multicolumn{1}{c}{$\begin{array}{c}\text { Bosque, Edad (años) } \\
\text { y zona de vida }\end{array}$} & \multicolumn{1}{c}{$\begin{array}{c}\text { Clasificación de Suelos } \\
\text { (Orden y Subgrupos) }\end{array}$}
\end{tabular}

Las zonas de vida son: bs-T: precipitación anual: $1100-1500 \mathrm{~mm}$, temperatura media $24-28^{\circ} \mathrm{C}$, estación seca de hasta 6.5 meses. bh-PV: precipitación anual: $1500-1950 \mathrm{~mm}$, temperatura media $24-28^{\circ} \mathrm{C}$, estación seca de $3.5-5$ meses.

mayor a 100 años que pudieron estar sometidos a la extracción maderera selectiva en el pasado. Las edades de los sitios fueron obtenidas de los registros históricos del PNSR y la HPA.

Establecimiento de las parcelas: Se establecieron 130 parcelas de muestreo de $10 \times 50 \mathrm{~m}$ (500 $\mathrm{m}^{2}$ o $0.05 \mathrm{ha}$ ) con un área total de 6.5 ha muestreadas, orientadas con rumbo norte. Se establecieron 100 parcelas en cinco bosques secundarios $(10,15,20,40$ y 60 años) (20 por sitio) y 30 parcelas en tres bosques maduros (>100 años) (10 por sitio), cuya forma rectangular es adecuada para medir el efecto del paisaje edáfico a pequeña escala (estudios $<1 \mathrm{ha}$ ) sobre la diversidad de especies arbóreas (Clark 2002). Otros detalles sobre la instalación de las parcelas se encuentran en Leiva et al. (2008). Cada parcela fue georeferenciada y en la misma se tomaron datos de paisaje como fisiografía local, porcentaje de pendiente, dirección de pendiente, rocosidad, drenaje, erosión, presencia de nivel freático y altitud.

Muestreo de la vegetación arbórea: Las parcelas se dividieron en cinco subparcelas de $10 \times 10 \mathrm{~m}$, donde se midió el diámetro a $1.3 \mathrm{~m}$ de altura (dap) de todos los individuos con dap $\geq 5 \mathrm{~cm}$ (árboles), lo que incluyó arbustos, árboles jóvenes y árboles maduros presentes en los estratos medio y superior del bosque (Apéndice 1). Además, se midieron individuos jóvenes (latizales, dap $\geq 1 \mathrm{y}<5 \mathrm{~cm}$ ) en el estrato inferior utilizando dos subparcelas de $1 \mathrm{x}$ $25 \mathrm{~m}$ en los extremos Suroeste y Noreste de la parcela principal (Apéndice 1). Se registró el dap $(\mathrm{cm})$ usando cinta diamétrica para la categoría árboles y pie de rey para la categoría 
latizales (Condit 1998). Se midió la altura (m) con un tubo telescópico para alturas $<15 \mathrm{~m}$ y un hipsómetro Haga para árboles $\geq 15 \mathrm{~m}$. El muestreo se realizó una vez, entre agosto del 2003 y noviembre del 2004. El índice de valor de importancia (IVI) se calculó como la sumatoria de los valores parciales (\%) del área basal, la densidad y la frecuencia de cada especie respecto al valor total en cada bosque.

Análisis estadístico: El análisis del efecto del tipo de suelo sobre el bosque se realizó examinando cada bosque por separado, pues cada uno es resultado de una serie única de procesos ecológicos, perturbaciones naturales, gradientes ambientales, geoformas y eventos de impacto humano. Se analizó la influencia de las clases de suelo sobre las características estructurales del bosque y la diversidad de especies arbóreas, comparando el área basal promedio $\left(\mathrm{m}^{2} \mathrm{ha}^{-1}\right)$, según la sumatoria del área basal $\left(\pi / 4 \cdot[\text { diámetro] }]^{2}\right)$ de todos los árboles medidos en cada parcela, la densidad promedio de individuos (No. individuosha ${ }^{-1}$ ), y la altura dominante $(\mathrm{m})$, que se obtuvo como el promedio de la altura del $10 \%$ de los individuos más altos en cada parcela, en todas las clases de suelo. Se realizó un análisis de varianza de un factor (Statistica versión 5.5, Statsoft Inc. 2000) estableciendo la clase de suelo y la edad como las fuentes de variación principal. En cada bosque se probó la hipótesis nula de que la clase de suelo y la edad no tienen un efecto importante sobre la estructura del bosque (densidad, altura y área basal). Además, se calculó el total de especies arbóreas presentes (riqueza) en cada sitio y clase de suelo utilizando EstimateS (versión 7.5, Colwell 2005), y la diversidad de especies según el índice de Shannon-Wiener (H'). Se probó la hipótesis nula de valores de diversidad iguales entre clases de suelos utilizando la prueba de $\mathrm{t}$ (Zar 1999). Este índice es recomendado para muestras $<1000$ individuos (Condit 1998). En cada sitio y clase de suelo se calcularon curvas de acumulación de riqueza (número de especies) y de diversidad de especies arbóreas (índice $\alpha$ de Fisher). El último índice es ampliamente utilizado por su habilidad discriminante entre sitios, no depende del tamaño de la muestra y no es afectado por las especies raras o las especies dominantes (Magurran 1988). Finalmente, se comparó el grado de similitud de especies arbóreas asociadas a las diferentes clases de suelos, utilizando el índice "Jaccard" (Chao et al. 2005). Este índice emplea un enfoque probabilístico basado en la abundancia (número de individuos) de las especies en común entre parcelas con suelos diferentes, sin tomar en cuenta las especies "no vistas". Esto reduce el sesgo al comparar muestras con tamaños distintos y con especies raras. Los cálculos se hicieron con un orden de muestreo con reemplazo.

\section{RESULTADOS}

Suelos dominantes: Se identificaron 17 clases de suelos pertenecientes a seis órdenes, siete subórdenes y ocho grandes grupos (6.5 ha); las intergradaciones (20 años) y extragradaciones (10 años) generaron 14 subgrupos de suelos (Leiva et al. 2008). Se encontraron al menos dos suelos dominantes en todos los bosques, y en un bosque maduro se identificaron tres suelos (Cuadro 1). Los Entisoles y los Vertisoles poco profundos fueron los órdenes más comunes, mostraron algunas características del material parental, y un desarrollo incipiente de los horizontes. Dentro de los Entisoles, el subgrupo más común (Ustorthents) se presentó en cuatro de los ocho sitios $(15,20,60$ y $>100$ años), con variaciones en la profundidad de la roca $(22-64 \mathrm{~cm})$ y con características ándicas (origen volcánico). Los Alfisoles, Inceptisoles, Ultisoles y Mollisoles presentaron buen drenaje en general y se encontraron sobre ignimbritas menos consolidadas y de relieve ondulado (Leiva et al. 2008).

\section{Estructura y diversidad según la edad} del bosque: La densidad de individuos, el área basal por hectárea y la altura dominante aumentaron con la edad del bosque, y alcanzaron valores similares a los observados en los bosques maduros a partir de los 40-60 años de sucesión (Prueba de t, p=0.65, Cuadro 2, Fig. 
CUADRO 2

Densidad de individuos ( $N / h a)$, área basal $\left(\mathrm{m}^{2} / \mathrm{ha}\right.$ ) y altura dominante ( $\mathrm{m}$ ) en BTS de edades crecientes, según el tamaño de los individuos (latizales y árboles) y suelos dominantes

TABLE 2

Density of individuals ( $N / h a)$, basal area $\left(\mathrm{m}^{2} / \mathrm{ha}\right)$ and dominant height (m) in TDF of increasing age, according to size class (saplings and trees) and dominant soil type

\begin{tabular}{|c|c|c|c|c|c|c|c|}
\hline \multirow{2}{*}{$\begin{array}{l}\text { Edad y } \\
\text { Suelo }\end{array}$} & \multirow[b]{2}{*}{$\mathrm{n}$} & \multicolumn{3}{|c|}{ Latizales (dap $\geq 1 \mathrm{y}<5 \mathrm{~cm}$ ) } & \multicolumn{3}{|c|}{ Árboles $($ dap $\geq 5$ cm $)$} \\
\hline & & $\begin{array}{l}\text { Densidad } \\
(\mathrm{N} / \mathrm{ha})\end{array}$ & $\begin{array}{c}\text { Área Basal } \\
\left(\mathrm{m}^{2} / \mathrm{ha}\right)\end{array}$ & $\begin{array}{l}\text { Altura } \\
(\mathrm{m})\end{array}$ & $\begin{array}{c}\text { Densidad } \\
(\mathrm{N} / \mathrm{ha})\end{array}$ & $\begin{array}{c}\text { Área Basal } \\
\left(\mathrm{m}^{2} / \mathrm{ha}\right)\end{array}$ & $\begin{array}{l}\text { Altura } \\
(\mathrm{m})\end{array}$ \\
\hline $\begin{array}{c}10 \\
\mathrm{a}\end{array}$ & 12 & $\begin{array}{c}2783 \pm 660 \\
(0-8000)\end{array}$ & $\begin{array}{c}1.37 \pm 0.44 \\
(0-5.60)\end{array}$ & $\begin{array}{c}2.94 \pm 0.36 \\
(0-4.68)\end{array}$ & $\begin{array}{c}500 \pm 80 \\
(160-1180)\end{array}$ & $\begin{array}{c}2.33 \pm 0.37 \\
(0.6-5.0)\end{array}$ & $\begin{array}{c}5.90 \pm 0.25 \\
(4.4-7.0)\end{array}$ \\
\hline b & 8 & $\begin{array}{c}4400 \pm 1009 \\
(400-8800)\end{array}$ & $\begin{array}{l}2.52 \pm 0.66 \\
(0.20-5.04)\end{array}$ & $\begin{array}{l}3.43 \pm 0.23 \\
(2.38-4.28)\end{array}$ & $\begin{array}{c}685 \pm 130 \\
(160-1320)\end{array}$ & $\begin{array}{c}3.62 \pm 0.84 \\
(0.7-8.5)\end{array}$ & $\begin{array}{c}5.79 \pm 0.43 \\
(4.1-7.6)\end{array}$ \\
\hline $\begin{array}{c}15 \\
\mathrm{a}\end{array}$ & 13 & $\begin{array}{c}6308 \pm 951 \\
(2600-12200)\end{array}$ & $\begin{array}{l}2.56 \pm 0.40 \\
(1.08-5.75)\end{array}$ & $\begin{array}{l}4.01 \pm 0.23 \\
(2.58-5.12)\end{array}$ & $\begin{array}{l}1155 \pm 112 \\
(520-1780)\end{array}$ & $\begin{array}{c}8.4 \pm 1.0 \\
(2.9-18.2)\end{array}$ & $\begin{array}{c}8.53 \pm 0.77 \\
(6.31-15.68)\end{array}$ \\
\hline $\mathrm{b}$ & 7 & $\begin{array}{c}7286 \pm 1243 \\
(2800-11600)\end{array}$ & $\begin{array}{l}2.85 \pm 0.44 \\
(1.60-4.86)\end{array}$ & $\begin{array}{l}4.04 \pm 0.24 \\
(2.86-4.81)\end{array}$ & $\begin{array}{l}1266 \pm 135 \\
(980-2000)\end{array}$ & $\begin{array}{c}6.63 \pm 0.69 \\
(4.6-9.6)\end{array}$ & $\begin{array}{l}7.32 \pm 0.50 \\
(4.73-8.91)\end{array}$ \\
\hline $\begin{array}{c}20 \\
\mathrm{a}\end{array}$ & 12 & $\begin{array}{c}5183 \pm 992 \\
(1600-14000)\end{array}$ & $\begin{array}{l}1.99 \pm 0.37 \\
(0.41-3.72)\end{array}$ & $\begin{array}{l}4.14 \pm 0.20 \\
(2.83-5.18)\end{array}$ & $\begin{array}{c}993 \pm 109 \\
(120-1600)\end{array}$ & $\begin{array}{c}7.3 \pm 1.1 \\
(0.5-16.5)\end{array}$ & $\begin{array}{c}7.32 \pm 0.53 \\
(3.88-11.37)\end{array}$ \\
\hline b & 8 & $\begin{array}{l}3900 \pm 677 \\
(600-6800)\end{array}$ & $\begin{array}{l}2.07 \pm 0.34 \\
(0.14-0.33)\end{array}$ & $\begin{array}{l}3.77 \pm 0.24 \\
(2.59-4.44)\end{array}$ & $\begin{array}{c}973 \pm 86 \\
(640-1280)\end{array}$ & $\begin{array}{c}6.76 \pm 0.74 \\
(4.7-10.1)\end{array}$ & $\begin{array}{l}7.33 \pm 0.33 \\
(5.80-8.52)\end{array}$ \\
\hline $\begin{array}{c}40 * \\
\mathrm{a}\end{array}$ & 11 & $\begin{array}{l}3964 \pm 804 \\
(200-9200)\end{array}$ & $\begin{array}{l}1.87 \pm 0.52 \\
(0.20-6.14)\end{array}$ & $\begin{array}{l}4.68 \pm 0.32 \\
(2.75-6.00)\end{array}$ & $\begin{array}{c}1085 \pm 51 \\
(860-1340)\end{array}$ & $\begin{array}{c}22.0 \pm 1.8 \\
(13.7-35.5)\end{array}$ & $\begin{array}{c}13.24 \pm 0.42 \\
(10.69-16.20)\end{array}$ \\
\hline $\mathrm{b}$ & 9 & $\begin{array}{c}3844 \pm 653 \\
(2200-7800)\end{array}$ & $\begin{array}{l}1.91 \pm 0.25 \\
(0.82-3.13)\end{array}$ & $\begin{array}{l}5.47 \pm 0.36 \\
(4.01-7.44)\end{array}$ & $\begin{array}{c}998 \pm 54 \\
(860-1380)\end{array}$ & $\begin{array}{c}20.7 \pm 1.6 \\
(15.3-27.1)\end{array}$ & $\begin{array}{c}16.10 \pm 0.51 \\
(14.55-19.00)\end{array}$ \\
\hline $\begin{array}{c}60 * \\
\mathrm{a}\end{array}$ & 11 & $\begin{array}{l}1782 \pm 292 \\
(200-3200)\end{array}$ & $\begin{array}{l}1.19 \pm 0.30 \\
(0.07-3.09)\end{array}$ & $\begin{array}{l}5.57 \pm 0.49 \\
(2.82-7.32)\end{array}$ & $\begin{array}{l}676 \pm 31 \\
(500-820)\end{array}$ & $\begin{array}{l}20.6 \pm 2.7 \\
(9.5-42.6)\end{array}$ & $\begin{array}{c}18.33 \pm 0.82 \\
(13.64-23.00)\end{array}$ \\
\hline $\mathrm{b}$ & 9 & $\begin{array}{l}3156 \pm 676 \\
(800-7400)\end{array}$ & $\begin{array}{l}1.41 \pm 0.28 \\
(0.57-3.08)\end{array}$ & $\begin{array}{l}4.93 \pm 0.39 \\
(3.82-7.42)\end{array}$ & $\begin{array}{c}1127 \pm 72 \\
(660-1320)\end{array}$ & $\begin{array}{c}24.7 \pm 1.6 \\
(17.6-34.3)\end{array}$ & $\begin{array}{l}15.70 \pm 0.64 \\
(13.26-19.67)\end{array}$ \\
\hline $\begin{array}{c}>100 * \\
\mathrm{a}\end{array}$ & 6 & $\begin{array}{c}2733 \pm 329 \\
(1800-4000)\end{array}$ & $\begin{array}{l}1.45 \pm 0.11 \\
(1.09-1.91)\end{array}$ & $\begin{array}{l}5.70 \pm 0.18 \\
(5.10-6.20)\end{array}$ & $\begin{array}{c}997 \pm 70 \\
(740-1200)\end{array}$ & $\begin{array}{c}26.7 \pm 4.3 \\
(15.1-43.4)\end{array}$ & $\begin{array}{c}17.03 \pm 0.86 \\
(13.38-18.88)\end{array}$ \\
\hline $\mathrm{b}$ & 4 & $\begin{array}{c}3500 \pm 238 \\
(3000-4000)\end{array}$ & $\begin{array}{l}1.71 \pm 0.33 \\
(1.06-2.58)\end{array}$ & $\begin{array}{l}5.57 \pm 0.47 \\
(4.50-6.77)\end{array}$ & $\begin{array}{l}715 \pm 55 \\
(620-860)\end{array}$ & $\begin{array}{c}17.5 \pm 2.4 \\
(12.8-21.9)\end{array}$ & $\begin{array}{c}21.1 \pm 2.2 \\
(16.3-26.8)\end{array}$ \\
\hline $\mathrm{c}$ & 6 & $\begin{array}{c}4100 \pm 583 \\
(3000-6000)\end{array}$ & $\begin{array}{l}1.43 \pm 0.25 \\
(0.88-2.44)\end{array}$ & $\begin{array}{l}5.40 \pm 0.27 \\
(4.43-5.85)\end{array}$ & $\begin{array}{c}930 \pm 16 \\
(900-980)\end{array}$ & $\begin{array}{l}22.0 \pm 4.0 \\
(9.7-33, .7)\end{array}$ & $\begin{array}{c}18.3 \pm 1.2 \\
(14.8-21.0)\end{array}$ \\
\hline d & 4 & $\begin{array}{c}3033 \pm 264 \\
(2400-3800)\end{array}$ & $\begin{array}{l}1.87 \pm 0.21 \\
(1.45-2.34)\end{array}$ & $\begin{array}{l}5.79 \pm 0.30 \\
(5.00-6.64)\end{array}$ & $\begin{array}{c}860 \pm 92 \\
(640-1040)\end{array}$ & $\begin{array}{l}23.5 \pm 5.5 \\
(8.6-34.2)\end{array}$ & $\begin{array}{c}18.5 \pm 1.2 \\
(15.0-22.2)\end{array}$ \\
\hline $\mathrm{e}$ & 4 & $\begin{array}{c}3450 \pm 377 \\
(2400-4200)\end{array}$ & $\begin{array}{l}1.93 \pm 0.15 \\
(1.61-2.30)\end{array}$ & $\begin{array}{l}5.91 \pm 0.60 \\
(4.33-7.25)\end{array}$ & $\begin{array}{c}780 \pm 91 \\
(560-980)\end{array}$ & $\begin{array}{c}44.5 \pm 9.4 \\
(32.5-72.1)\end{array}$ & $\begin{array}{c}21.56 \pm 0.79 \\
(20.25-23.40)\end{array}$ \\
\hline $\mathrm{f}$ & 4 & $\begin{array}{c}3500 \pm 545 \\
(2400-5000)\end{array}$ & $\begin{array}{l}1.85 \pm 0.43 \\
(1.21-3.06)\end{array}$ & $\begin{array}{l}5.26 \pm 0.35 \\
(4.54-6.20)\end{array}$ & $\begin{array}{c}1040 \pm 62 \\
(900-1180)\end{array}$ & $\begin{array}{c}36.4 \pm 3.3 \\
(29.0-44.5)\end{array}$ & $\begin{array}{c}18.32 \pm 1.97 \\
(13.96-23.00)\end{array}$ \\
\hline $\mathrm{g}$ & 2 & $\begin{array}{l}2300 \pm 1300 \\
(1000-3600)\end{array}$ & $\begin{array}{l}1.28 \pm 0.54 \\
(0.74-1.82)\end{array}$ & $\begin{array}{l}5.31 \pm 0.61 \\
(4.70-5.91)\end{array}$ & $\begin{array}{c}900 \pm 20 \\
(880-920)\end{array}$ & $\begin{array}{c}26.3 \pm 4.7 \\
(21.6-31.0)\end{array}$ & $\begin{array}{c}17.46 \pm 2.16 \\
(15.30-19.63)\end{array}$ \\
\hline
\end{tabular}

Los valores son promedios \pm ES; los valores mínimos y máximos aparecen entre paréntesis. n: Número de parcelas establecidas en cada subgrupo de suelo. dap: diámetro a $1.3 \mathrm{~m}$ sobre el suelo. * Sitios con diferencias en una o más variables. Medias diferentes significativamente entre suelos señaladas en cursiva. 
1). La máxima densidad de latizales y de árboles se presentó en el bosque de 15 años $(6650 \pm 744$ y $1194 \pm 86$ individuosha $^{-1}$, respectivamente), a partir del cual la densidad, especialmente de los latizales, disminuyó hasta valores promedio de $3247 \pm 177$ y $893 \pm 31$ latizales y árboles ha $^{-1}$, respectivamente, en los bosques maduros (Fig. 1A). El área basal $(G)$ de los latizales alcanzó un valor máximo en el bosque de 15 años $\left(2.66 \pm 0.30 \mathrm{~m}^{2} \mathrm{ha}^{-1}\right)$ y luego disminuyó hasta valores similares al promedio de los bosques maduros (1.64 $\pm 0.10 \mathrm{~m}^{2} \mathrm{ha}^{-1}$, Fig. 1B). El área basal de los árboles aumentó con la edad, hasta alcanzar valores de $27.8 \pm 2.4 \mathrm{~m}^{2} \mathrm{ha}^{-1}$ en los bosques maduros. La altura dominante de los latizales y los árboles se incrementó con la edad, hasta alcanzar valores de $5.60 \pm 0.13 \mathrm{~m}$ y $18.85 \pm 0.56 \mathrm{~m}$, respectivamente, en los bosques maduros (Fig. 1C). Estos resultados coinciden con otros estudios de la dinámica de regeneración del BTS (Ruiz et al. 2005)

La diversidad de latizales $\left(\mathrm{H}^{\prime}=3.10 \pm 0.15\right)$ y árboles $\left(\mathrm{H}^{\prime}=3.73 \pm 0.11\right)$ aumentó hasta alcanzar valores máximos en los bosques maduros (Cuadro 3). En los bosques secundarios más jóvenes (10, 15 y 20 años) los valores de H' de los latizales fueron mayores que los de los árboles (Cuadro 3), pero este comportamiento se invirtió a partir de los 40 años, lo que resultó en mayor riqueza de especies en los bosques maduros de mayor edad. Si se considera el tipo de suelo (Cuadro 3), no hubo diferencias importantes en la diversidad H' de los latizales en los bosques de 10, 15, 20 y 40 años, pero en el bosque de 60 años hubo mayor diversidad $(\mathrm{p}=0.002)$ en el suelo modal $a$ (Ultisol, Typic Haplustults) que en el $b$ (Entisol, Typic Ustorthents). Las diferencias en $\mathrm{H}^{\prime}$ de los árboles según el tipo de suelo fueron más frecuentes, y se presentaron en los bosques de $10(p=0.05), 15(p=0.01)$ y 60 años $(\mathrm{p}=0.05)$. El bosque de 60 años presentó una diversidad $\mathrm{H}$ ' de latizales y árboles similares a los valores de los bosques maduros ( $\mathrm{p}>$ 0.44). Los valores de H' de los bosques maduros no presentaron diferencias importantes entre las diferentes clases de suelo ( $p>0.37$ ) (Cuadro 3). En los bosques maduros hubo

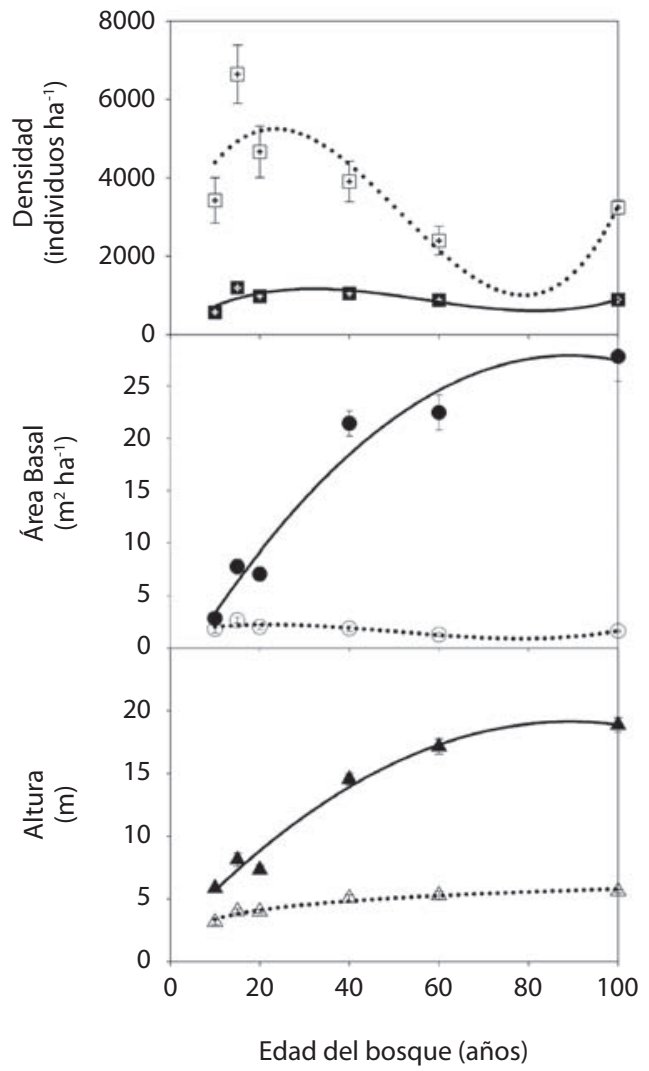

Fig. 1. Densidad, área basal y altura dominante en BTS en sucesión en Santa Rosa, Costa Rica. Promedio \pm error estándar por edad y clase de tamaño: latizales (dap 1-5 $\mathrm{cm}$, símbolos abiertos, línea punteada) y árboles (dap $\geq 5$ $\mathrm{cm}$, símbolos rellenos, línea continua). $\mathrm{n}=20$ en bosques jóvenes y 30 en bosques maduros ( $>100$ años). Las funciones de mejor ajuste para latizales y árboles, respectivamente, son: Densidad, $y=0.0093 x^{3}-1.5796 x^{2}+72.719 x$ $+155.84\left(\mathrm{r}^{2}=0.49\right) ; y=0.0494 x^{3}-7.5942 x^{2}+274.41 x+$ $2358.7\left(\mathrm{r}^{2}=0.60\right)$. Área basal, $y=0.00001 x^{3}-0.0022 x^{2}+$ $0.0759 x+1.5041\left(\mathrm{r}^{2}=0.69\right) ; y=-0.0039 x^{2}+0.699 x-3.23$ $\left(\mathrm{r}^{2}=0.96\right)$. Altura dominante, $y=1.0506 * \ln (x)+0.9396$ $\left(\mathrm{r}^{2}=0.96\right) ; y=-0.0021 x^{2}+0.3825 x+2.03\left(\mathrm{r}^{2}=0.98\right)$.

Fig. 1. Density, basal area and dominant height of successional TDF forests in Santa Rosa, Costa Rica. Mean \pm standard error for age and size class: saplings (dbh 1-5 $\mathrm{cm}$, open symbols, dotted line) and trees ( $\mathrm{dbh} \geq 5 \mathrm{~cm}$, filled symbols, solid line). $\mathrm{n}=.20$ for young forests and 30 for mature forests ( $>100$ years). Best-fit models for saplings and trees are: Density, $y=0.0093 x^{3}-1.5796 x^{2}+72.719 x$ $+155.84\left(\mathrm{r}^{2}=0.49\right) ; y=0.0494 x^{3}-7.5942 x^{2}+274.41 x+$ $2358.7\left(\mathrm{r}^{2}=0.60\right)$. Basal area, $y=0.00001 x^{3}-0.0022 x^{2}+$ $0.0759 x+1.5041\left(\mathrm{r}^{2}=0.69\right) ; y=-0.0039 x^{2}+0.699 x-3.23$ $\left(\mathrm{r}^{2}=0.96\right)$. Dominant height, $y=1.0506 * \ln (x)+0.9396$ $\left(\mathrm{r}^{2}=0.96\right) ; y=-0.0021 x^{2}+0.3825 x+2.03\left(\mathrm{r}^{2}=0.98\right)$. 
CUADRO 3

Número de individuos (Ind.), riqueza de especies (Esp.) y diversidad, por edad del bosque y clase de suelo según el indice Shannon-Wiener ( $\left.H^{\prime}\right)$, en BTS de edades crecientes en Santa Rosa, Costa Rica

TABLE 3

Density of individuals $\left(N \mathrm{ha}^{-1}\right)$, species richness (Esp) and Shannon-Wiener diversity index (H') according to forest age and soil type, in TDF of increasing age in Santa Rosa, Costa Rica

\begin{tabular}{|c|c|c|c|c|c|c|c|c|}
\hline \multirow{2}{*}{$\begin{array}{l}\text { Edad y Suelo } \\
\text { (años) }\end{array}$} & \multicolumn{4}{|c|}{ Latizales (dap 1-5 cm) } & \multicolumn{4}{|c|}{ Árboles (dap $\geq 5 \mathrm{~cm}$ ) } \\
\hline & Ind. & Esp. & $\mathrm{H}^{, *}$ & $\mathrm{DE}$ & Ind. & Esp. & $\mathrm{H}^{*} *$ & $\mathrm{DE}$ \\
\hline 10 & 346 & 16 & $2.09 \mathrm{c}$ & 0.14 & 574 & 15 & $1.83 \mathrm{c}$ & 0.02 \\
\hline $\mathrm{a}$ & 160 & 14 & 1.99 & 0.20 & 300 & 13 & 1.85 & 0.03 \\
\hline $\mathrm{b}$ & 168 & 10 & 1.82 & 0.23 & 274 & 11 & 1.75 & 0.04 \\
\hline 15 & 457 & 20 & $2.25 \mathrm{bc}$ & 0.10 & 1183 & 22 & $1.95 \mathrm{c}$ & 0.07 \\
\hline a & 284 & 19 & 2.25 & 0.15 & 734 & 16 & 2.00 & 0.10 \\
\hline $\mathrm{b}$ & 169 & 12 & 1.89 & 0.12 & 452 & 11 & 1.69 & 0.07 \\
\hline 20 & 366 & 18 & $2.47 \mathrm{~b}$ & 0.08 & 991 & 22 & $2.02 \mathrm{c}$ & 0.09 \\
\hline a & 219 & 17 & 2.30 & 0.17 & 595 & 18 & 1.96 & 0.11 \\
\hline $\mathrm{b}$ & 144 & 12 & 2.35 & 0.11 & 387 & 12 & 1.94 & 0.18 \\
\hline 40 & 324 & 32 & $2.86 \mathrm{a}$ & 0.07 & 1044 & 59 & $3.11 \mathrm{~b}$ & 0.10 \\
\hline $\mathrm{a}$ & 194 & 23 & 2.72 & 0.08 & 592 & 44 & 3.05 & 0.12 \\
\hline $\mathrm{b}$ & 142 & 23 & 2.60 & 0.14 & 447 & 34 & 2.79 & 0.10 \\
\hline 60 & 229 & 34 & $2.87 \mathrm{a}$ & 0.14 & 858 & 65 & $3.60 \mathrm{a}$ & 0.09 \\
\hline $\mathrm{a}$ & 95 & 20 & 2.87 & 0.14 & 362 & 53 & 3.46 & 0.06 \\
\hline $\mathrm{b}$ & 141 & 19 & 2.22 & 0.16 & 494 & 49 & 3.20 & 0.12 \\
\hline$>100^{w}$ & 492 & 47 & $3.10 \mathrm{a}$ & 0.15 & 1346 & 91 & $3.73 \mathrm{a}$ & 0.11 \\
\hline $\mathrm{E}+\mathrm{I}$ & 109 & 18 & 2.22 & 0.35 & 273 & 48 & 3.17 & 0.27 \\
\hline A & 227 & 34 & 2.75 & 0.15 & 608 & 59 & 3.35 & 0.13 \\
\hline M & 120 & 18 & 2.31 & 0.15 & 439 & 54 & 3.45 & 0.15 \\
\hline
\end{tabular}

${ }^{*}$ Letras distintas entre filas indican diferencias significativas en el dato total de $\mathrm{H}$ ' por edad $(\mathrm{P}<0.05)$. DE: Desviación estándar de H' (según EstimateS). Para bosques maduros: ${ }^{\text {w }}$ datos totales; E+I, A, M, indican respectivamente datos para Entisoles+Inceptisoles, Alfisoles y Mollisoles.

mayor diversidad en los Alfisoles que en los Mollisoles y los Entisoles.

Efecto del suelo sobre la estructura y la diversidad del bosque: El tipo de suelo no tuvo influencia importante sobre la estructura del bosque, especialmente a nivel de los latizales. Los casos en que hubo diferencias estuvieron concentrados en los árboles, en los bosques de 40, 60 y 100 años (Cuadro 2). En el bosque de 40 años, la altura de los árboles en el suelo Andic Dystrustepts fue significativamente mayor $(\mathrm{p}<0.001)$ que la altura de los árboles del Entic Haplustolls. En el bosque de 60 años 
hubo diferencias significativas en la densidad de árboles $(\mathrm{p}<0.0001)$ y la altura dominante del dosel $(p=0.03)$. El suelo menos desarrollado (Typic Ustorthents) sostuvo mayor densidad de árboles y menor altura del dosel que el suelo más desarrollado (Typic Haplustults, Cuadro 2). Asimismo, en los modales a y b de los bosques maduros se observaron diferencias significativas en la densidad de los árboles $(\mathrm{p}=0.02)$, mayor en el suelo menos profundo (Lithic Haplustolls) que en el más profundo (Entic Haplustolls) (Cuadro 2).

La acumulación de riqueza de especies fue sostenida y no se estabilizó a lo largo de la cronosecuencia de regeneración, y fue consistentemente mayor en los bosques maduros (Fig. 2A, 2B). Si se consideran muestras menores a 100 individuos en cada curva y en ambas clases diamétricas (latizales y árboles), la acumulación de riqueza de especies para los bosques secundarios más jóvenes (10, 15 y 20 años) fue muy similar. Esta "similitud" en las curvas volvió a presentarse en bosques secundarios más viejos (40 y 60 años) y en los bosques maduros (> 100 años). Si se consideran muestras mayores a 100 individuos, la acumulación de riqueza fue más individualizada en cada uno de los bosques muestreados.

La acumulación de diversidad (índice $\alpha$ de Fisher) reveló diferencias importantes entre las clases de suelo descritas dentro de cada bosque (Fig. 3). En los bosques de menor edad (10, 15 y 20 años) la diversidad fue mayor en los latizales (Fig. 3), mientras que a partir de 40 años la diversidad de árboles fue mayor. Este comportamiento también se evidenció con el índice de Shannon-Wiener H' (Cuadro 3). En todos los bosques examinados, la clase de suelo se relacionó con diferencias importantes en la acumulación de diversidad de especies arbóreas. La excepción fue el bosque de 20 años donde las curvas no presentaron diferencias según la clase de suelo (Fig. 3). En los bosques maduros la mayor acumulación de riqueza se presentó en los Alfisoles, caracterizados por menor fertilidad natural que los suelos del orden Mollisoles (Fig. 3; ver Leiva et al. 2008).

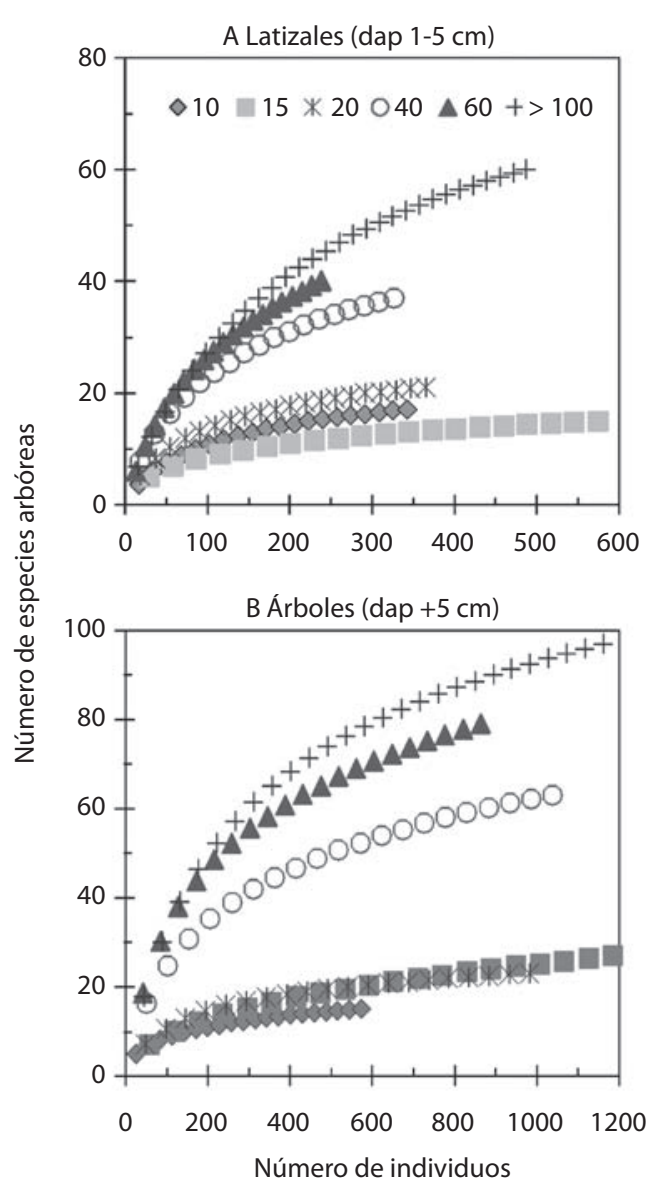

Fig. 2. Curvas de acumulación de riqueza de especies en BTS de edades crecientes en Santa Rosa, Costa Rica. Datos totales para cada edad del bosque en años $(\bullet 10$, $\mathbf{1 5}$, * 20, ○ 40, $\boldsymbol{\Delta} 60$ y + >100) y tamaño de los individuos (A = latizales, $\mathrm{B}=$ árboles).

Fig. 2. Species richness accumulation curves (Colwell 2005) in TDF of increasing age in Santa Rosa, Costa Rica. Total data for forest sites, age in years $(\bullet 10, \mathbf{1 5}, * 20$, O $40, \boldsymbol{\Delta} 60 \mathrm{y}+>100)$ and size class (A =saplings, $\mathrm{B}=$ trees).

Similitud de especies según la edad y el suelo: En comparación con el índice JaccardChao, el índice Jaccard clásico indicó menor similitud de especies entre clases de suelos diferentes (Fig. 4). Sin embargo, considerando ambos índices, la similitud de los latizales disminuyó conforme aumentó la edad del bosque. Si se considera el índice Jaccard-Chao solamente, 


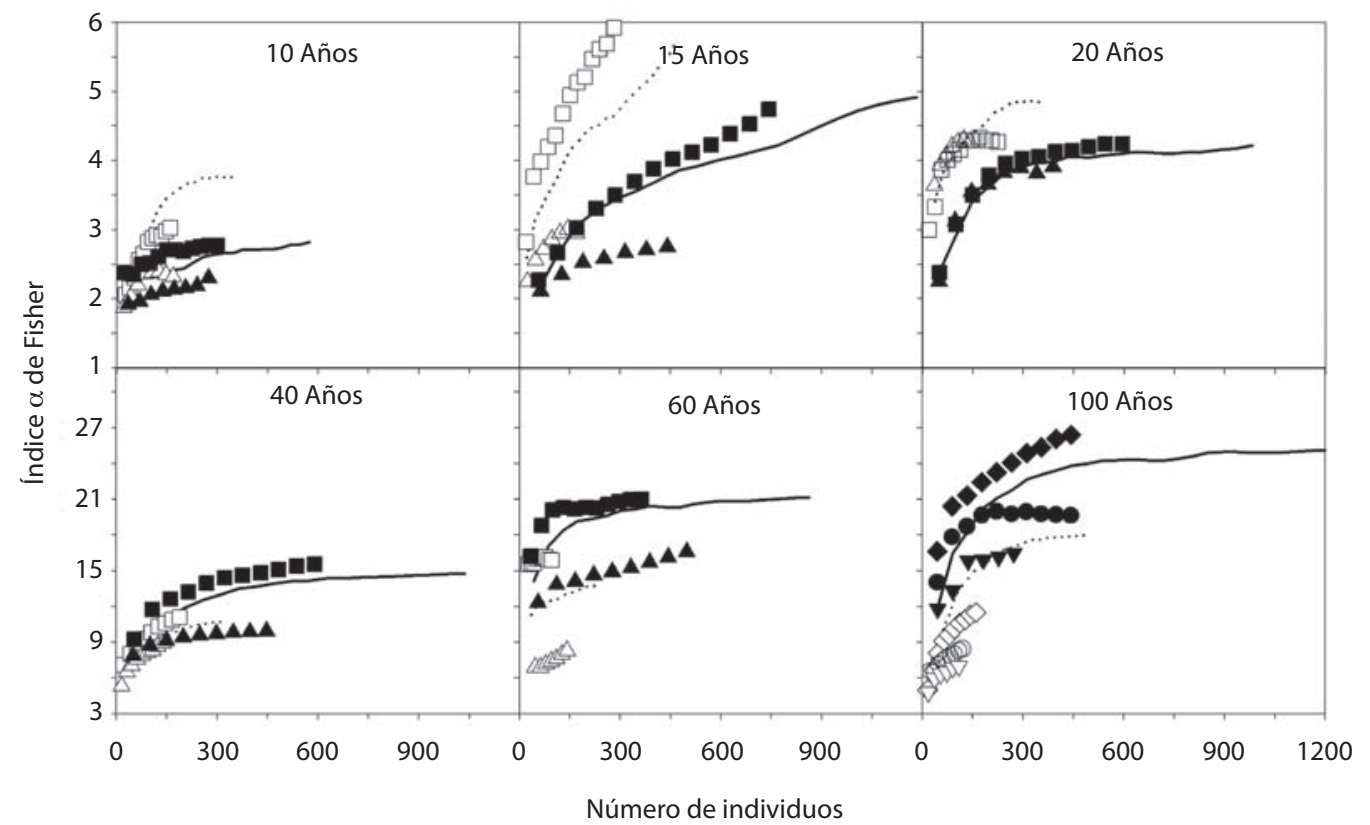

Fig. 3. Curvas de acumulación de diversidad de especies arbóreas (índice $\alpha$ de Fisher), en BTS de edades crecientes en Santa Rosa, Costa Rica. Símbolos vacíos y rellenos indican las clases de tamaño latizales y árboles, respectivamente. Las líneas muestran los datos totales por edad: --- latizales, y — árboles. Los símbolos indican la clase de suelo en bosques jóvenes ( $\square$ suelo a; $\triangle \boldsymbol{\Delta}$ suelo b). En bosques maduros (>100 años), los símbolos indican los datos para suelos del orden Alfisoles $(\diamond \diamond)$, Mollisoles $(\bigcirc \bullet)$, y Entisoles + Inceptisoles $(\nabla \nabla)$.

Fig. 3. Tree species diversity curves (Fisher's $\alpha$ ), in TDF of increasing age in Santa Rosa, Costa Rica. Open symbols show data for saplings and filled symbols show data for trees. Lines represent total data for each forest age: --- saplings, and trees. Symbols for young sites show soil class ( $\square \mathbf{D}$ soil a; $\triangle \boldsymbol{\Delta}$ soil b). In mature forests ( $>100$ years), symbols show data for different soil orders: Alfisols $(\diamond \diamond)$, Mollisols $(\bigcirc \bullet)$, and Entisols + Inceptisols $(\nabla \nabla)$.

la menor similitud de latizales (0.46) se presentó al comparar los Alfisoles y los Mollisoles de los bosques maduros. Para los árboles, el índice Jaccard clásico no mostró respuesta a la edad del bosque. Por el contrario, se observó alta similitud entre las clases de suelo en los bosques secundarios jóvenes de 10, 15 y 20 años (índice Jaccard-Chao 0.89-0.97), con una leve disminución a valores de similitud 0.69-0.79 a partir de los 60 años. El aumento en riqueza de especies pareció estar relacionado con la disminución de la similitud. Una vez más, el bosque de 60 años mostró valores semejantes a los bosques maduros, con un valor del índice Jaccard-Chao de 0.69 para los árboles.

\section{DISCUSIÓN}

La sucesión del Bosque Tropical Seco: La sucesión del BTS se diferenció en varios aspectos y pareció tener más estados sucesionales que la sucesión de los bosques lluviosos, debido probablemente a las mayores restricciones fisonómicas y fisiológicas experimentadas en estos ecosistemas estacionalmente secos. Las limitaciones al crecimiento impuestas por la sequía, y los retrasos en la sucesión causados por la periodicidad del fuego y otras perturbaciones como "El Niño", resultaron en una secuencia sucesional compleja, caracterizada por pulsos estacionales de recursos como el 


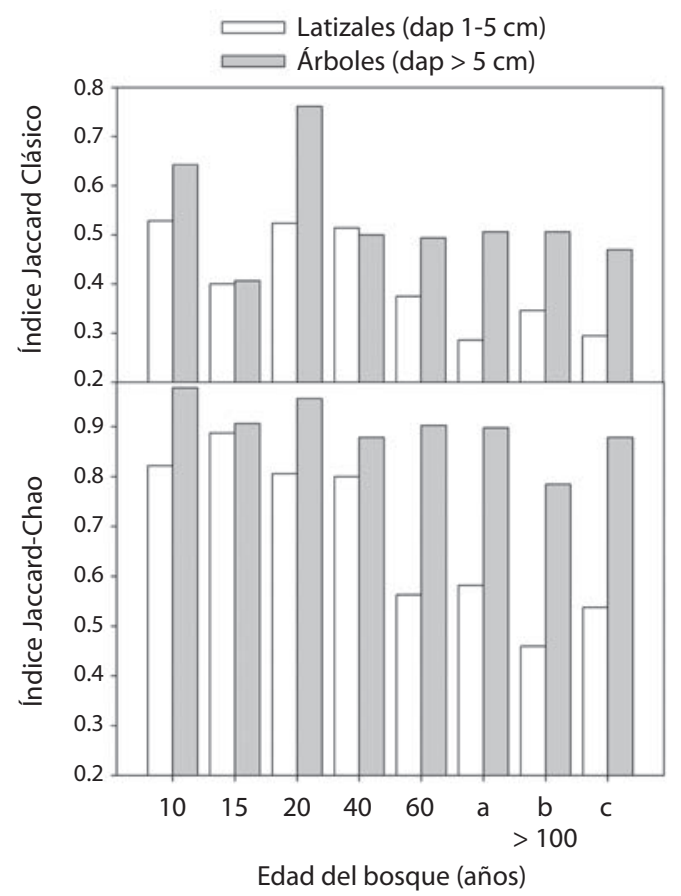

Fig. 4. Similitud de especies arbóreas calculada con los índices de Jaccard clásico y Jaccard-Chao, según la edad del bosque y la clase de suelo, en BTS de edades crecientes en Santa Rosa, Costa Rica. En bosques maduros ( $>100$ años) se compara la similitud entre a) Alfisoles y Entisoles, b) Alfisoles y Mollisoles, y c) Mollisoles y Entisoles + Inceptisoles.

Fig. 4. Tree species similarity estimated with Jaccard classic and Jaccard-Chao indexes, according to forest age and soil class in TDF of increasing age in Santa Rosa, Costa Rica. In mature forests ( $>100$ years), similarity is compared for a) Alfisols and Entisols, b) Alfisols and Mollisols, c) Mollisols and Entisols + Inceptisols.

agua y los nutrimentos, y transiciones fenológicas muy marcadas en la vegetación.

La flora sucesional del BTS estuvo constituida por comunidades florísticamente más simples y menos diversas que las de los bosques lluviosos (Apéndice 1), y la frecuencia de la reproducción asexual entre las plantas pioneras causó un proceso de regeneración dominado inicialmente por grupos de individuos clonales, con distribución irregular del área foliar. El área basal de los BTS maduros fue comparable a la de los bosques lluviosos, pero se diferenció por la alta contribución de pocos individuos de gran diámetro y copa amplia, lo que posiblemente refleja las consecuencias de la sequía estacional sobre la supervivencia de las plántulas, y resulta en mayor distancia entre los individuos.

La recuperación del BTS ocurrió en edades de sucesión temprana a intermedia (40-60 años), a partir de las cuales la estructura y la diversidad arbóreas fueron similares a las observadas en los BTS maduros. Sin embargo, la acumulación de riqueza de especies fue sostenida y alcanzó un valor asintótico, y fue consistentemente mayor en los bosques maduros (Fig. 3). La riqueza de árboles estimada con el índice de Shannon-Wiener y las curvas de rarefacción de diversidad ( $\alpha$ de Fisher) aumentaron con la sucesión, mientras que los índices de similitud Jaccard-Chao (diversidad $\beta$ ) disminuyeron. Esto coincide con otros estudios (Ewel 1977, 1980) que concluyeron que aunque la recuperación de la estructura y las funciones del bosque puede ocurrir rápidamente, la recuperación de la riqueza de especies es un proceso mucho más lento.

Diversidad de los suelos y sus efectos sobre la estructura del bosque: Se describieron suelos pertenecientes a seis órdenes: Entisoles, Vertisoles, Alfisoles, Mollisoles, Ultisoles e Inceptisoles, cuya diversidad pareció ser consecuencia de varios factores. Los sitios planos generaron un proceso de acumulación localizada de agua y nutrimentos, y el clima estacional y la presencia de ignimbritas propició la formación de Vertisoles y Entisoles (Buol et al. 1989). Los suelos clasificados como Inceptisoles, Mollisoles y Ultisoles se desarrollaron en paisajes ondulados, generando meteorización en la pendiente y acumulación de materiales en los microvalles. Esta heterogeneidad topográfica y físico-química del suelo a escalas pequeñas ha sido implicada en el control de la distribución y la abundancia de las plantas, pero es difícil evaluar su efecto sobre la vegetación, dada la importancia de los factores no edáficos y las limitaciones estadísticas 
asociadas a la abundancia de especies raras en los bosques tropicales (Clark 2002).

En este sentido, y particularmente entre los latizales, no se encontró un efecto importante del suelo sobre la estructura de los bosques. Las diferencias más importantes se presentaron en los árboles (diámetro $\geq 5 \mathrm{~cm}$ ) al comparar órdenes de suelos diferentes. Este fue el caso de los Entisoles recientes y poco profundos en comparación con los Ultisoles más viejos, arcillosos y poco fértiles del bosque de 60 años, y también en el bosque de 40 años al comparar Inceptisoles poco fértiles con un Mollisol muy fértil. En los bosques más jóvenes ( $<20$ años), la mayor diversidad de latizales obedeció a la mayor densidad de individuos en esta clase y a la mayor probabilidad de encontrar especies "nuevas" en el inventario arbóreo (Condit et al. 1998). Esta tendencia cambió a partir de los 40 años, cuando la cantidad de árboles sobrepasó la de diámetros menores, y las especies heliófitas más longevas comenzaron a dominar los estratos superiores (Apéndice 1).

Efectos del suelo sobre la diversidad de las especies: A escala mundial, la diversidad y riqueza de especies (diversidad $\beta$ ) están determinadas por factores climáticos y geomorfológicos, grandes perturbaciones como los huracanes, procesos biogeográficos, y patrones de especiación y extinción (Leigh et al. 2004, Clark 2002). En Costa Rica, la mayoría de los estudios sobre las relaciones suelo-planta se han realizado en bosques húmedos (Huston 1980, Herrera \& Finegan 1997, Clark 2002), donde la distribución no aleatoria causada por los factores edáficos son poco evidentes y de magnitud baja (Clark 2002). Esta tendencia se ha repetido en otros estudios con helechos (Tuomisto y Ruokolainen 1994), palmas (Kahn 1987) y árboles (Condit et al. 1998). En los bosques maduros evaluados, los suelos de menor fertilidad presentaron índices de diversidad $\alpha$ de Fisher mayores. Esto concuerda con otros estudios en los que la diversidad de especies vegetales ha sido mayor en condiciones restrictivas y de alta competencia, que permiten la coexistencia de especies con tasas de crecimiento disímiles, o que pueden causar la distribución preferencial de especies con hábitos fenológicos contrastantes, que difieren en sus estrategias de utilización de los recursos (Goldberg 1982). En el BTS de Santa Rosa, la distribución no aleatorias de Quercus oleoides (roble encino, Montoya-Maquin 1966), y Crescentia alata (jícaro, Alvarado et al. 1982) sugieren la existencia de asociaciones edáficas específicas. Sin embargo, el número de especies que muestran preferencias edáficas parece ser reducido y típicamente menor al 20\% (Pitman et al. 1999, 2001). Esto ha sido evidente en suelos de baja fertilidad como los Oxisoles y los Espodosoles del Amazonas (Duivenvoorden 1996, Clark 2002, Leigh et al. 2004) y en los Alfisoles y Entisoles examinados en este estudio (Leiva et al. 2008).

Similitud según la edad del bosque y la clase de suelo: Considerando todos los sitios, la probabilidad de encontrar especies de árboles compartidas entre clases de suelo diferentes (índice Jaccard-Chao 0.69-0.97) fue alta. En los bosques más jóvenes con suelos planos y poco profundos $(20-60 \mathrm{~cm})$, no se observó un efecto del suelo en la distribución de las especies, por lo que la similitud fue mayor. El aumento en la diversidad de árboles con la edad y la mayor incidencia de especies raras $(<1$ individuoha $^{-1}$, Apéndice 1), pudo haber generado una composición florística más heterogénea, y una disminución en la similitud entre las clases de suelos. Al analizar los latizales, la similitud Jaccard-Chao disminuyó hasta alcanzar valores mínimos en los bosques maduros. En los bosques maduros, la menor similitud se presentó al comparar la colección de especies de los Alfisoles y los Mollisoles, y fue consecuencia de la mayor diversidad encontrada en los bosques con suelos menos fértiles y con mayores restricciones físicas. Diversos estudios han concluido que suelos más restrictivos permiten el desarrollo de ecosistemas más diversos (Huston 1980, Clark 2002).

Los análisis de correlación efectuados en los Entisoles ( $<60 \mathrm{~cm}$ profundidad) revelaron un agotamiento temprano de los cationes $(\mathrm{Ca}$, 
$\mathrm{Mg}, \mathrm{K}, \mathrm{Na}$ y $\mathrm{CIC}$ ) durante los primeros 20 años de regeneración boscosa, que más tarde se recuperaron en los bosque maduros. En contraste, el contenido de materia orgánica no varió significativamente a lo largo de la cronosecuencia sucesional examinada, lo que contradice los resultados de otros estudios en sitios aledaños. Los incrementos sustanciales en la abundancia de macroporos y en la conductividad hidráulica a lo largo de la cronosecuencia, fueron probablemente el resultado de la mayor biomasa y mortalidad estacional de las raíces en sitios más viejos. Efectivamente, la asignación de biomasa a diferentes compartimentos de la vegetación (raíces, tallos, hojas y material reproductivo) cambia durante la sucesión. La biomasa foliar es producida más rápido primero, seguida por la producción de tallos, pero más tarde ocurre un aumento en la producción de raíces con un ciclo de vida muy dinámico (Ewel 1971). Como consecuencia del alto reciclaje anual de la biomasa radical causado por la sequía, la abundancia de mesoporos disminuyó y la de macroporos aumentó con la sucesión, paralelamente con la conductividad hidráulica del suelo. Consecuentemente, el agua disponible para las plantas disminuyó con la sucesión. Estos cambios en las propiedades físicas del suelo pueden tener importantes consecuencias sobre la fenología de las plantas durante la sucesión (Gerhardt \& Hytteborn 1992, Wright et al. 2001, Turnbull et al. 2005) (Apéndice 1). Las especies dominantes en los bosques maduros podrían tener que lidiar con sequías más severas y mayores riesgos de cavitación (Hacke et al. 2000, Brodribb et al. 2003), que podrían ser contrarrestados mediante el desarrollo de sistemas radicales extensivos y la producción de follaje capaz de soportar estrés hídrico más severo (Brodribb et al. 2003, Villalobos-Rodríguez 2001). Sin embargo, a pesar de la alta variación edáfica y fenológica identificada en los BTS de Santa Rosa (Villalobos-Rodríguez 2001), los árboles del BTS son predominantemente generalistas en relación al suelo, tolerantes a la heterogeneidad de los sitios, y con poca especialización en relación con la heterogeneidad edáfica.

\section{AGRADECIMIENTOS}

Este trabajo fue financiado por el Programa de Ecología Terrestre de la Fundación Andrew W. Mellon. Agradecemos al personal del Parque Nacional Santa Rosa, especialmente a Róger Blanco y a María Marta Chavarría.

\section{RESUMEN}

Se estudió la sucesión del bosque tropical seco (BTS) de Santa Rosa, Guanacaste, Costa Rica, en cinco sitios boscosos secundarios y tres bosques maduros. Se analizó el efecto de la clase de suelo sobre la estructura del bosque y la diversidad de especies arbóreas. Los ocho sitios sucesionales estaban localizados a lo largo de una cronosecuencia de regeneración $(10,15,20,40,60$ and >100 años) en la que se identificaron 17 pedones y seis órdenes de suelos con moderada a alta fertilidad, clasificados como Entisoles y Vertisoles, aunque los Mollisoles, Alfisoles, Inceptisoles y Ultisoles estuvieron presentes también. Se establecieron 130 parcelas de 10 por $50 \mathrm{~m}$ ( 0.05 ha), 20 en cada bosque secundario y 10 en cada bosque antiguo ( 6.5 ha en total), de acuerdo con la topografía. Se midió el diámetro a la altura de pecho (dap) de todos los individuos presentes en cada parcela, la altura dominante y el área basal $\left(\mathrm{m}^{2} \mathrm{ha}^{-1}\right)$, usando dos poblaciones: latizales (dap $\geq 1 \mathrm{y}<5 \mathrm{~cm}$ ) y árboles (dap $\geq 5 \mathrm{~cm}$ ). Cada bosque presentó al menos dos pedones de suelo bien diferenciados. La alta variación edáfica encontrada se debió posiblemente al origen piroclástico de la meseta ignimbrítica de Santa Rosa, las variaciones en los tiempos de deposición del material parental, y los patrones de erosión. A excepción de dos sitios, no se presentaron diferencias importantes en la estructura del bosque entre las clases de suelos examinadas, según la densidad de individuos, el área basal y la altura dominante. La diversidad de especies arbóreas medida según el índice Shannon-Wiener y las curvas de rarefacción construidas con el índice $\alpha$ de Fisher, presentaron diferencias considerables entre las clases de suelos. Esta tendencia se explicó por la influencia de las características edáficas sobre las distribuciones no aleatorias de las especies arbóreas, la escala de medición del estudio, la forma de la parcela, y el muestreo sistemático utilizado. En general, los suelos de menor fertilidad presentaron mayor riqueza de especies, indicando que los sitios más restrictivos redujeron la competencia y permitieron la coexistencia de individuos con tasas de crecimiento disímiles. Los cambios en las propiedades físicas encontrados a lo largo de una cronosecuencia de Entisoles indicaron que los árboles podrían experimentar estrés hídrico más severo conforme la sucesión avanza, lo que podría requerir ajustes en el comportamiento fenológico y en las propiedades ecofisiológicas de las especies dominantes. En general, nuestros resultados sugieren que la especialización edáfica es más pronunciada en BTS maduros, y que la mayoría de las especies del BTS son generalistas en relación con la 
clase de suelo, altamente tolerantes a la heterogeneidad de los sitios, y muestran poca especialización fisiológica para responder a la variabilidad edáfica en distancias cortas.

Palabras clave: Parque Nacional Santa Rosa, bosque seco tropical, bosques secundarios, bosques maduros, regeneración arbórea, estructura, diversidad, génesis de suelos, curvas de rarefacción, índices de similitud.

\section{REFERENCIAS}

Alvarado, A., A. Stam \& S. Redhead. 1982. Crescentia alata (Jícaro) and vertisol distribution in burned savannah in Guanacaste, Costa Rica, p. 245-247. In B. Williamson (ed.). Tropical Biology: an ecological approach. University of Miami, Miami, EEUU.

Bazzaz, F.A. 1979. The physiological ecology of plant succession. Ann. Rev. Ecol. Syst. 10: 351-371.

Bazzaz, F.A. \& S.T.A. Pickett. 1980. Physiological ecology of tropical succession: a comparative review. Ann. Rev. Ecol. Syst. 11: 287-310.

Brodribb, T.J., N.M. Holbrook \& M.V. Gutiérrez. 2003. Relations between stomatal closure, leaf turgor and xylem vulnerability in eight tropical dry forest trees. Plant Cell Environ. 26: 443-450.

Buol, S.W., F.D. Hole \& R.J. McCraken. 1989. Soil Genesis and Classification. Iowa State University, Iowa, EEUU.

Chao, A., R.L. Chazdon, R.K. Colwell \& T.J. Shen. 2005. A new statistical approach for assessing similarity of species composition with incidence and abundance data. Ecol. Let. 8: 148-159.

Chazdon, R.L. 2003. Tropical forest recovery: legacies of human impact and natural disturbances. Perspect. Plant. Ecol. 6: 51-71.

Clark, D.B., M.W. Palmer \& D.A. Clark. 1999. Edaphic factors and the landscape-scale distributions of tropical rain forest trees. Ecology 80: 2662-2675.

Clark, D.B. 2002. Los factores edáficos y la distribución de las plantas, p. 193-221. In M.R. Guariguata \& G.H. Kattan (eds.). Ecología y conservación de bosques neotropicales. LUR, Cartago, Costa Rica.

Colwell, R.K. 2005. EstimateS versión 7.5: statistical estimation of species richness and shared species from samples. University of Connecticut, EEUU. (Disponible en http://viceroy.eeb.uconn.edu/estimates)

Condit, R. 1998. Tropical forest census plots: methods and results from Barro Colorado Island, Panama and a comparison with other plots. Springer, Berlin, Alemania.

Daubenmire, R. 1972. Ecology of Hyparrhenia rufa (Ness) in derived savanna in north-western Costa Rica. J. Appl. Ecol. 9: 11-23.

Duivenvoorden, J.F. 1996. Patterns of tree species richness in rain forest of the middle Caquetárea, Colombia, NW Amazonia. Biotropica 28: 142-158.

Eamus, D. \& L. Prior. 2001. Ecophysiology of trees of seasonally dry tropics: comparisons among phenologies. Adv. Ecol. Res. 32: 113-197.

Ewel, J. 1971. Biomass changes in early tropical succession. Turrialba 21: 110-112.

Ewel, J. 1977. Differences between wet and dry successional tropical ecosystems. Geo-Eco-Trop 1: 103117.

Ewel, J. 1980. Tropical succession: manifold routes to maturity. Biotropica 12: 2-7.

Gerhardt, K. \& H. Hytteborn. 1992. Natural dynamics and regeneration methods in tropical dry forests -an introduction. J. Veg. Sci. 3: 361-364.

Goldberg, D.E. 1982. The distribution of evergreen and deciduous trees relative to soil type: an example from the Sierra Madre, México, and a general model. Ecology 63: 942-951.

Hacke, U.G., J.S. Sperry, B.E. Ewers, D.S. Ellsworth, K.V.R. Schäfer \& R. Oren. 2000. Influence of soil porosity on water use in Pinus taeda. Oecologia 124: 495-505.

Herrera, B. \& B. Finegan. 1997. Substrate conditions, foliar nutrients and the distributions of two canopy tree species in a Costa Rican secondary rain forest. Plant and Soil 191: 259-267.

Holbrook, N.M., J.L. Whitbeck \& H.A. Mooney. 1995. Drought responses of neotropical dry forest trees, $\mathrm{p}$. 243-276. In S.H. Bullock, H.A. Mooney \& E. Medina (eds.). Seasonally dry tropical forest. Cambridge, Reino Unido.

Holdridge, LR. 1978. Ecología basada en zonas de vida. IICA, San José, Costa Rica.

Huston, M. 1980. Soil nutrients and tree species richness in Costa Rican forests. J. Biogeogr. 7: 147-157.

Janzen, H. 1986. Guanacaste National Park: Tropical ecological and cultural restoration. EUNED, San José, Costa Rica. 
Janzen, H. 1988. Management of habitat fragments in a tropical dry forest: growth. Ann. Mo. Bot. Gard. 75: 105-116.

Kahn, F. 1987. The distribution of palms as a function of local topography in Amazonian terra-firme forests. Experientia 43: 251-259.

Kramer, E.A. 1997. Measuring landscape changes in remnant tropical dry forest, p. 386-399. In W.F. Lawrence \& R.G. Bierregaard (eds.). Tropical forest remnants: ecology, management, and conservation of fragmented communities. University of Chicago Press, Chicago, EEUU.

Lathwell, D.J. \& T.L. Grove. 1986. Soil-plant relationships in the tropics. Ann. Rev. Ecol. Syst. 17: 1-16.

Leigh Jr., E.G., P. Davidar, C.W. Dick, J.P. Puyravaud, J. Terborgh, H. ter Steege \& S.J. Wright. 2004. Why do some tropical forests have so many species of trees? Biotropica 36: 447-473.

Leiva, J.A., R. Mata, O.J. Rocha \& M.V. Gutiérrez. 2009 Cronología de la regeneración del bosque tropical seco en Santa Rosa, Costa Rica. I. Características edáficas. Rev. Biol. Trop. 57: 801-815.

Maas, J.M. 1995. Conversion of tropical dry forest to pasture and agriculture, p. 399-422. In S.H Bullock, H.A. Mooney \& E. Medina (eds.). Seasonally dry tropical forests. Cambridge University, Cambridge, Reino Unido.

Magurran, A.E. 1988. Ecological diversity and its measurement. Princeton, Nueva Jersey, EEUU.

Mesquita, R.C.G., K. Ickes, G. Ganade \& G.B. Williamson. 2001. Alternative successional athways in the Amazon Basin. J. Ecol. 89: 528-537.

Montoya-Maquín, J.M. 1966. Notas fitogeográficas sobre el Quercus oleoides Cham y Schlecht. Turrialba 16: $57-66$.

Mulkey, S.S, R.L. Chazdon \& A.P. Smith. 1996. Tropical forest plant ecophysiology. Chapman \& Hall, Nueva York, EEUU.

Murphy, P.G. \& A.E. Lugo. 1995. Dry forests of Central America and the Caribbean, p. 9-34. In S.H Bullock, H.A. Mooney \& E. Medina (eds.). Seasonally dry tropical forests. Cambridge University, Cambridge, Reino Unido.

Pitman, N.C.A, J. Terborgh, M.R. Silman \& P. Núñez. 1999. Tree species distribution in an upper Amazonian forest. Ecology 80: 2651-2661.
Pitman, N.C.A, J. Terborgh, M.R. Silman, P. Núñez, D.A. Neil, C.E. Cerón, W.A. Palacios \& M. Sulestia. 2001. Dominance and distribution of tree species in upper Amazonian terra firme forest. Ecology 82: 2101-2117.

Rico-Gray, V. \& J.G. García-Franco. 1992. Vegetation and soil seed bank of successional stages in tropical lowland deciduous forest. J. Veg. Sci. 3: 617-624.

Ruiz, J., M.C. Fandiño \& R.L. Chazdon. 2005. Vegetation structure, composition, and species richness across a 56-year chronosequence of dry tropical forest on Providencia Island, Colombia. Biotropica 37: 520-530.

Russell, A.E., J.W. Raich, O.J. Valverde-Barrantes \& R.F. Fisher. 2007. Tree species effects on soil properties in experimental plantations in tropical moist forest. Soil Sci. Soc. Am. J. 71: 1389-1397.

Smith, C.K., H.L. Gholz \& F. de Assis. 1998. Soil nitrogen dynamics and plant-induced soil changes under plantations and primary forest in lowland Amazonia, Brazil. Plant and Soil 200: 193-204.

StatSoft Inc. 2000. Statistica 5.5 for Windows [Computer program manual]. StatSoft, Tulsa, EEUU.

Stemmermann, L. \& T. Ishle. 1993. Replacement of Metrosideros polymorpha, 'hi'a, in Hawaiian dry forest succession. Biotropica 25: 36-45.

Turnbull, M.H., D.T. Tissue, K.L. Griffin, S.J. Richardson, D.A. Peltzer \& D. Whitehead. 2005. Respiration characteristics in temperate rainforest tree species differ along a long-term soil-development chronosequence. Oecologia 143: 271-279.

Tuomisto, H. \& K. Ruokolainen. 1994. Distribution of Pteridophyta and Melastomataceae along an edaphic gradient in an Amazonian rain forest. J. Veg. Sci. 5: 25-34.

Villalobos-Rodríguez, R. 2001. Fenología y relaciones hídricas de los árboles de un fragmento de bosque seco neotropical. Tesis de Licenciatura, Universidad de Costa Rica. San José, Costa Rica.

Wright, I.J., P.B. Reich \& M. Westoby. 2001. Strategy shifts in leaf physiology, structure and nutrient content between species of high- and low-rainfall and high- and low-nutrient habitats. Funt. Ecol. 15: 423-434.

Zar, J.H. 1999. Biostatistical analysis. Prentice-Hall, Nueva Jersey, EEUU.

Zinke, P.J. 1962. The pattern of influence of individual forest trees on soil properties. Ecology 43: 130-133. 


\section{APÉNDICE I}

Especies e Indices de Valor de Importancia (IVI) a lo largo de una crono-secuencia sucesional en el Bosque Tropical Seco de Santa Rosa, Costa Rica

\begin{tabular}{|c|c|c|c|c|c|c|c|c|c|}
\hline \multirow[t]{2}{*}{ Familia } & \multirow[t]{2}{*}{ Género y Especie } & \multirow[t]{2}{*}{ Autoridad } & \multicolumn{6}{|c|}{ Edad (años) } & \multirow{2}{*}{$\begin{array}{l}\text { IVI } \\
\text { Total }\end{array}$} \\
\hline & & & 10 & 15 & 20 & 40 & 60 & $>100$ & \\
\hline \multirow[t]{4}{*}{ Anacardiaceae } & Astronium graveolens & Jacq. & & & & & 5 & 19 & 23.8 \\
\hline & Spondias mombin & $\mathrm{L}$. & & & & 7 & 9 & 11 & 26.8 \\
\hline & Spondias purpurea & $\mathrm{L}$. & & & & 2 & 1 & & 3.4 \\
\hline & Spondias radlkoferi & Donn. Sm. & & & & & & 1 & 1.4 \\
\hline \multirow[t]{4}{*}{ Annonaceae } & Annona purpurea & Moc. \& Sesse ex Dunal & & & & & 1 & 5 & 5.4 \\
\hline & Annona reticulata & L. & & & & & 2 & 3 & 4.8 \\
\hline & Desmopsis bibracteata & (Rob.) Safford & & & & 1 & 2 & 2 & 3.9 \\
\hline & Sapranthus palanga & R. E. Fr. & & & & 4 & 5 & 2 & 10.7 \\
\hline \multirow[t]{2}{*}{ Apocynaceae } & Stemmadenia obovata & $\begin{array}{l}\text { (Hook. Y Arn.) K. } \\
\text { Schum. }\end{array}$ & & & & 18 & 3 & 7 & 27.9 \\
\hline & Thevetia ovata & (Cav.) A. DC. & & & & 0.3 & & & 0.3 \\
\hline Araliaceae & Sciadodendron excelsum & Griseb. & & & & 0 & & 1 & 1.0 \\
\hline Arecaceae & Bactris guineensis & (L.) H.E. Moore & & & & 4 & & & 4.4 \\
\hline \multirow[t]{3}{*}{ Asteraceae } & Cromolaena glaberrima & $\begin{array}{l}\text { (DC.) R. M. King \& H. } \\
\text { Rob. }\end{array}$ & & 1 & & & & & 0.7 \\
\hline & Lasianthaea fruticosa & (L.) K.M. Becker & & & & 1 & 0.3 & & 0.9 \\
\hline & Verbesina gigantea & Jacq. & & 1 & & & & & 0.7 \\
\hline \multirow[t]{5}{*}{ Bignoniaceae } & Crescentia alata & Kunth & 5 & 1 & 5 & & & & 10.0 \\
\hline & Crescentia cujete & L. & & 1 & & & & & 1.4 \\
\hline & Tabebuia ochracea & (Cham.) Standl. & & 1 & & 23 & 8 & 15 & 47.0 \\
\hline & Tabebuia rosea & (Vertol.) DC. & & 1 & 1 & & & & 1.3 \\
\hline & Xylophragma seemannianum & (Kunth) Sandw. & & & & 1 & & & 1.3 \\
\hline Bixaceae & Cochlospermum vitifolium & (Willd.) Spreng. & 60 & 3 & 56 & 13 & 4 & 4 & 139.9 \\
\hline Bombacaceae & Pseudobombax septenatum & (Jacq.) Dugand & & & & & & 1 & 0.6 \\
\hline \multirow[t]{6}{*}{ Boraginaceae } & Bourreria quirosii & Standl. & & & & 0.3 & & & 0.3 \\
\hline & Cordia alliodora & (Ruiz \& Pav.) Cham. & & 1 & & 9 & 3 & 4 & 16.4 \\
\hline & Cordia collococca & $\mathrm{L}$. & & & & & & 1 & 0.5 \\
\hline & Cordia dentata & Poir. & & & & & & 1 & 0.5 \\
\hline & Cordia inermis & (Mill.) I.M. Johnst. & 2 & 6 & 11 & 1 & & & 20.8 \\
\hline & Cordia panamensis & Riley & & & & 1 & 6 & 5 & 11.7 \\
\hline \multirow[t]{2}{*}{ Burseraceae } & Bursera graveolens & (Kunth) Triana \& Planch. & & & 10 & 3 & 2 & 4 & 19.1 \\
\hline & Bursera simarouba & $\mathrm{L}$. & & 1 & 3 & 6 & 8 & 14 & 32.0 \\
\hline \multirow[t]{2}{*}{ Capparidaceae } & Capparis frondosa & Jacq. & & & & & 2 & & 2.2 \\
\hline & Capparis indica & (L.) Fawc. y Renkle & & & & & 3 & & 3.4 \\
\hline Cecropiaceae & Cecropia peltata & $\mathrm{L}$. & & & & & 1 & 5 & 6.4 \\
\hline Celastraceae & Maytenus segoviarum & Standl. \& L. O. Williams & & & & & 4 & 2 & 6.1 \\
\hline
\end{tabular}




\section{APÉNDICE I (Continuación)}

\begin{tabular}{|c|c|c|c|c|c|c|c|c|c|}
\hline \multirow[t]{2}{*}{ Familia } & \multirow[t]{2}{*}{ Género y Especie } & \multirow[t]{2}{*}{ Autoridad } & \multicolumn{6}{|c|}{ Edad (años) } & \multirow{2}{*}{$\begin{array}{l}\text { IVI } \\
\text { Total }\end{array}$} \\
\hline & & & 10 & 15 & 20 & 40 & 60 & $>100$ & \\
\hline \multirow[t]{3}{*}{ Chrysobalanaceae } & Chrysobalanus icaco & L. & & & & & & 0.5 & 0.5 \\
\hline & Hirtella racemosa & Lam. & & & & & 4 & 71 & 74.8 \\
\hline & Licania arborea & Seem. & & & & & 4 & 29 & 32.1 \\
\hline Clusiaceae & Garcinia intermedia & (Pittier) Hammel & & & & & & 6 & 5.6 \\
\hline Dilleniaceae & Curatella americana & $\mathrm{L}$. & 13 & 12 & 8 & & & & 33.1 \\
\hline Ebenaceae & Diospyros salicifolia & Willd. & 5 & 5 & 3 & 1 & 1 & 1 & 16.8 \\
\hline Elaeocarpaceae & Sloanea terniflora & $\begin{array}{l}\text { (Moc. Y Sess, ex DC.) } \\
\text { Standl. }\end{array}$ & & & & & 2 & 17 & 18.5 \\
\hline Erythroxylaceae & Erythroxylum havanense & Jacq. & & & & 9 & 13 & & 21.4 \\
\hline \multirow[t]{10}{*}{ Euphorbiaceae } & Adelia triloba & (Mull. Arg) Hemsl. & & & & & & 3 & 2.5 \\
\hline & Bernardia nicaraguensis & Standl. & & & & 7 & 10 & 3 & 21.2 \\
\hline & Croton niveus & Jacq. & & & & 1 & & & 1.4 \\
\hline & Euphorbia schlechtendalii & Boiss & & & & 2 & & & 2.4 \\
\hline & Garcia nutans & Vahl & & & & & 1 & & 0.5 \\
\hline & Mabea montana & Müll. Arg. & & & & & 1 & & 0.6 \\
\hline & Margaritaria nobilis & L. f. & 2 & & & & & & 1.6 \\
\hline & Sapium glandulosum & (L.) Morong & & & & & 1 & 2 & 2.4 \\
\hline & Sapium sp. & & & & & & & 1 & 0.6 \\
\hline & Sebastiania pavoniana & Müll. Arg. & & & & & 8 & 13 & 21.2 \\
\hline Fabaceae & Bauhinia ungulata & $\mathrm{L}$. & & 1 & & 3 & 2 & 2 & 7.8 \\
\hline \multirow[t]{3}{*}{ Caesalpinaceae } & Hymenaea courbaril & $\mathrm{L}$. & & & & & 4 & 79 & 83.8 \\
\hline & Senna atomaria & Irwin \& Barneby & & 1 & & 3 & & & 3.5 \\
\hline & Senna skinneri & Benth. & & 1 & & & & & 0.7 \\
\hline Fabaceae & Acacia collinsii & Saff. & 4 & 23 & 14 & 7 & 4 & 5 & 57.8 \\
\hline \multirow[t]{9}{*}{ Mimosaceae } & Acacia cornigera & $\mathrm{L}$. & 1 & & & & & 1 & 2.7 \\
\hline & Acacia farnesiana & (L.) Willd. & 1 & & 2 & & & & 3.2 \\
\hline & Calliandra bijuga & Rose & & & & & 2 & & 1.5 \\
\hline & Enterolobium cyclocarpum & (Jacq.) Griseb. & & & & & 2 & & 1.7 \\
\hline & Inga vera & $\begin{array}{l}\text { Willd. Ssp spurea } \\
\text { (Willd.) J. León }\end{array}$ & & & & & & 1 & 0.6 \\
\hline & Lysiloma auritum & (Schltdl.) Benth. & & & & & 4 & 4 & 8.5 \\
\hline & Lysiloma divaricatum & (Jacq.) J.F. Macbr. & & 1 & 1 & 7 & 0.3 & & 8.9 \\
\hline & Piptadenia flava & (Spreng. ex DC.) Benth. & & & & 1 & & & 1.2 \\
\hline & Samanea saman & (Jacq.) Merr & & & & & 2 & & 1.9 \\
\hline Fabaceae & Acosmiun panamense & (Benth.) Yakovl. & & & & & 1 & & 0.8 \\
\hline \multirow[t]{4}{*}{ Papilionaceae } & Andira inermis & (Wright) Kunth & & & & & & 4 & 3.6 \\
\hline & Ateleia herbert-smithii & Pittier & & & & 0.3 & & & 0.3 \\
\hline & Dalbergia retusa & Hemsl. & 3 & 3 & 4 & 1 & 3 & 3 & 17.5 \\
\hline & Diphysa americana & (Mill.) M. Sousa & & & & & & 1 & 0.5 \\
\hline
\end{tabular}




\section{APÉNDICE I (Continuación)}

\begin{tabular}{|c|c|c|c|c|c|c|c|c|c|}
\hline \multirow[t]{2}{*}{ Familia } & \multirow[t]{2}{*}{ Género y Especie } & \multirow[t]{2}{*}{ Autoridad } & \multicolumn{6}{|c|}{ Edad (años) } & \multirow{2}{*}{$\begin{array}{l}\text { IVI } \\
\text { Total }\end{array}$} \\
\hline & & & 10 & 15 & 20 & 40 & 60 & $>100$ & \\
\hline & Gliricidia sepium & (Jacq.) Kunth ex Walp & 52 & 33 & 15 & 1 & 8 & & 110.0 \\
\hline & Lonchocarpus acuminatus & (Schltdl.) M. Sousa & & & & & & 3 & 2.6 \\
\hline & Lonchocarpus felipei & N. Zamora & & 1 & & 3 & 5 & 6 & 14.9 \\
\hline & Lonchocarpus guatemalensis & Benth. & & & & & & 1 & 0.9 \\
\hline & Lonchocarpus hughesii & M. Sousa & & & & 1 & & & 0.8 \\
\hline & Lonchocarpus minimiflorus & Donn. Sm. & & 1 & & 10 & 7 & & 17.8 \\
\hline & Lonchocarpus rugosus & Benth & & & & & & 1 & 1.5 \\
\hline & Lonchocarpus salvadorensis & Pittier & & & & 0.4 & & & 0.4 \\
\hline & Machaerium biovulatum & Micheli & & 3 & & 3 & 3 & 2 & 11.4 \\
\hline & Myrospermum frutescens & Jacq. & & & & 2 & & 1 & 2.9 \\
\hline & Swartzia cubensis & (Britton y Wilson) Standl. & & & & & 0.3 & 9 & 8.9 \\
\hline Fagaceae & Quercus oleoides & Schltdl \& Cham. & & 17 & 12 & & 1 & 14 & 44.9 \\
\hline \multirow[t]{6}{*}{ Flacourtiaceae } & Casearia corymbosa & Kunth & & & & & 0.3 & & 0.8 \\
\hline & Casearia praecox & Griseb. & & & & 3 & & 4 & 6.2 \\
\hline & Casearia sylvestris & Sw. & & & & & 11 & 20 & 31.3 \\
\hline & Casearia tremula & $\begin{array}{l}\text { (Griseb.) Griseb. ex W. } \\
\text { Wright }\end{array}$ & & & & 5 & 4 & 2 & 11.9 \\
\hline & Prockia crucis & P. Browne ex L. & & & & 1 & & & 1.0 \\
\hline & Zuelania guidonia & (Sw.) Britton \& Millsp. & & & 1 & 0.5 & 1 & 10 & 11.5 \\
\hline Hippocrateaceae & Semialarium mexicanum & (Miers) Mennega & 33 & 39 & 10 & 18 & 24 & 9 & 133.6 \\
\hline Lauraceae & Ocotea veraguensis & (Meissn.) Mez & & & & & 7 & 28 & 35.2 \\
\hline \multirow[t]{3}{*}{ Malpighiaceae } & Banisteriopsis cornifolia & (Kunth) B. L. Rob. & & 1 & 1 & & & & 2.0 \\
\hline & Byrsonima crassifolia & (L.) Kunth & 56 & 48 & 34 & 1 & & 1 & 139.6 \\
\hline & Malpighia glabra & $\mathrm{L}$. & & & & 0.5 & 0.3 & 2 & 2.7 \\
\hline \multirow[t]{2}{*}{ Melastomataceae } & Miconia argentea & (Sw.) DC. & & & & & & 5 & 5.3 \\
\hline & Mouriri myrtilloides & (Sw.) Poir & & & & & & 48 & 47.8 \\
\hline \multirow[t]{6}{*}{ Meliaceae } & Cedrela odorata & $\mathrm{L}$. & & & & 6 & & 1 & 6.5 \\
\hline & Guarea glabra & Vahl & & 1 & & & 1 & 8 & 8.8 \\
\hline & Swietenia macrophylla & King & 3 & 7 & 5 & & 1 & 1 & 17.2 \\
\hline & Trichilia americana & (Sees, y Moc.) T.D. Penn. & & & & & 1 & 2 & 2.5 \\
\hline & Trichilia hirta & $\mathrm{L}$. & & & & & & 1 & 0.5 \\
\hline & Trichilia martiana & C. DC. & & & & & 1 & 1 & 1.8 \\
\hline \multirow[t]{5}{*}{ Moraceae } & Brosimum alicastrum & Sw. & & & & & & 16 & 15.6 \\
\hline & Castilla elastica & Sessé & & & & & 0.3 & 28 & 28.2 \\
\hline & Picus ovalis & (Liebm.) Miq. & & & & & 1 & & 0.6 \\
\hline & Maclura tinctoria & (L.) G. Don & & & & 3 & 2 & 6 & 10.8 \\
\hline & Trophis racemosa & (L.) Urb. & & & & & & 4 & 3.6 \\
\hline Myrsinaceae & Ardisia revoluta & Kunth & & & & & 1 & 17 & 17.7 \\
\hline Myrtaceae & Eugenia acapulcensis & Steud & & & & & & 1 & 0.6 \\
\hline
\end{tabular}




\section{APÉNDICE I (Continuación)}

\begin{tabular}{|c|c|c|c|c|c|c|c|c|c|}
\hline \multirow[t]{2}{*}{ Familia } & \multirow[t]{2}{*}{ Género y Especie } & \multirow[t]{2}{*}{ Autoridad } & \multicolumn{6}{|c|}{ Edad (años) } & \multirow{2}{*}{$\begin{array}{l}\text { IVI } \\
\text { Total }\end{array}$} \\
\hline & & & 10 & 15 & 20 & 40 & 60 & $>100$ & \\
\hline & Eugenia hypargyrea & Standl. & & & & 1 & & 5 & 6.3 \\
\hline & Eugenia monticola & (Sw.) DC. & & & & 0.5 & 0.4 & 2 & 3.0 \\
\hline & Eugenia oerstedeana & O. Berg & & & & & & 24 & 23.5 \\
\hline & Eugenia salamensis & Donn. Sm. & & & & & & 3 & 3.1 \\
\hline & Psidium guajava & L. & 1 & & 1 & & & & 2.2 \\
\hline & Psidium sartorianum & (O. Berg) Nied. & & & & 1 & & & 0.6 \\
\hline Nyctaginaceae & Pisonea macranthocarpa & Donn. Sm. & & & & & & 1 & 0.5 \\
\hline Ochnaceae & Ouratea lucens & (Kunth) Engl. & & & & & 1 & 8 & 8.3 \\
\hline Opiliaceae & Agonandra macrocarpa & L.O. Williams & & & & 0.4 & & 1 & 0.9 \\
\hline \multirow[t]{2}{*}{ Polygonaceae } & Coccoloba guanacastensis & W.C. Burger & & & & & 0.3 & 1 & 1.1 \\
\hline & Coccoloba padiformis & Meisn. & & & & & & 1 & 0.8 \\
\hline Proteaceae & Roupala montana & Aubl. & & 1 & & & & 1 & 1.3 \\
\hline \multirow[t]{2}{*}{ Rhamnaceae } & Colubrina triflora & Brogn. & & & & & 0.3 & & 0.3 \\
\hline & Karwinskia calderonii & Standl. & & 1 & & & & & 0.8 \\
\hline \multirow[t]{15}{*}{ Rubiaceae } & Alibertia edulis & (Rich.) A. Rich. & & 2 & 1 & & 0.3 & 3 & 6.1 \\
\hline & Amaioua corymbosa & Kunth & & & & 2 & & & 1.6 \\
\hline & Calycophyllum candidissimum & (Vahl) DC. & & & & 1 & 4 & 4 & 8.7 \\
\hline & Chomelia spinosa & Jacq. & & & & 14 & 2 & 7 & 23.2 \\
\hline & Coutarea hexandra & (Jacq.) K. Schum. & & & & 0.3 & & & 0.3 \\
\hline & Exostema caribaeum & (Jacq.) Roem. y Schult. & & & & 1 & & & 0.7 \\
\hline & Exostema mexicanum & A. Grey. & & & & & 9 & 11 & 19.2 \\
\hline & Genipa americana & $\mathrm{L}$. & 16 & 6 & 9 & & 6 & 2 & 38.9 \\
\hline & Guettarda macrosperma & Donn. Sm. & & & & 3 & 9 & 3 & 15.4 \\
\hline & Hamelia patens & Jacq. & & & & & & 1 & 1.1 \\
\hline & Ixora floribunda & (A. Rich.) Griseb. & & & & & & 5 & 5.3 \\
\hline & Posoqueria latifolia & (Rudge) Roem. \& Schult. & & & & & & 1 & 0.7 \\
\hline & Randia monantha & Benth. & & & & 1 & 1 & 3 & 4.5 \\
\hline & Randia sp. & & & & & & 1 & & 0.6 \\
\hline & Randia thurberi & S. Watson & & & 2 & & & & 1.6 \\
\hline \multirow[t]{2}{*}{ Rutaceae } & Esenbeckia berlandieri & Baill. & & & & & 0.3 & & 0.3 \\
\hline & Zanthoxylum setulosum & P. Wilson & & & & & 0.3 & 1 & 0.9 \\
\hline \multirow[t]{4}{*}{ Sapindaceae } & Allophylus occidentalis & (Sw.) Radlk. & & & & 3 & 3 & 5 & 10.9 \\
\hline & Cupania guatemalensis & (Turcz) Radlk. & & & & & 1 & 10 & 11.2 \\
\hline & Dilodendron costaricense & $\begin{array}{l}\text { (Radlk.) A. Gentry y } \\
\text { Steyerm }\end{array}$ & & & & & & 8 & 8.2 \\
\hline & Thouinidium decandrum & (Humb. Y Bonpl.) Radlk & & & & 4 & 1 & 5 & 10.0 \\
\hline \multirow[t]{2}{*}{ Sapotaceae } & Chrysophyllum brenesii & Cronquist & & & & & 0.3 & 5 & 5.7 \\
\hline & Manilkara chicle & (Pittier) Gilly & & & & & 6 & 41 & 47.3 \\
\hline
\end{tabular}


APÉNDICE I (Continuación)

\begin{tabular}{|c|c|c|c|c|c|c|c|c|c|}
\hline \multirow[t]{2}{*}{ Familia } & \multirow[t]{2}{*}{ Género y Especie } & \multirow[t]{2}{*}{ Autoridad } & \multicolumn{6}{|c|}{ Edad (años) } & \multirow{2}{*}{$\begin{array}{l}\text { IVI } \\
\text { Total }\end{array}$} \\
\hline & & & 10 & 15 & 20 & 40 & 60 & $>100$ & \\
\hline \multirow[t]{3}{*}{ Simaroubaceae } & Picramnia antidesma & Sw. & & & & & & 24 & 23.8 \\
\hline & Quassia amara & L. & & & & & 0.3 & 23 & 23.6 \\
\hline & Simarouba glauca & DC. & 4 & & & 2 & 3 & 7 & 16.3 \\
\hline \multirow[t]{4}{*}{ Sterculiaceae } & Guazuma ulmifolia & Lam. & 2 & & 4 & 5 & 8 & 10 & 28.9 \\
\hline & Helicteres baruensis & Jacq. & & & 2 & 7 & 1 & 2 & 11.8 \\
\hline & Helicteres guazumifolia & Kunth & 4 & 5 & 7 & & & & 15.1 \\
\hline & Sterculia apetala & (Jacq.) Karst. & & & & & 2 & 7 & 8.8 \\
\hline Theophrastac. & Jacquinia nervosa & C. Presl & & & & 10 & 1 & 1 & 11.0 \\
\hline \multirow[t]{3}{*}{ Tiliaceae } & Apeaba tibourbou & Aubl. & & & & & & 6 & 6.4 \\
\hline & Luehea candida & (DC.) Mart. & & 12 & 13 & 33 & 15 & 48 & 120.8 \\
\hline & Luehea speciosa & Willd. & & & & & & 1 & 0.7 \\
\hline Turneraceae & Erblichia odorata & Seem. & & & & & & 4 & 4.1 \\
\hline Ulmaceae & Trema micrantha & (L.) Blume & 1 & & & & & & 1.1 \\
\hline \multirow[t]{3}{*}{ Verbenaceae } & Cornutia grandiflora & $\begin{array}{l}\text { (Schltdl. \& Cham.) } \\
\text { Schauer }\end{array}$ & & & & 2 & 1 & & 2.1 \\
\hline & Limpia oxyphyllaria & (Donn. Sm.) Standl. & & 2 & & & & & 20 \\
\hline & Redhera trinervis & (S.F. Blake) Moldenke & 32 & 58 & 65 & 12 & 12 & 6 & 185.1 \\
\hline
\end{tabular}

